UNIVERSIDADE DE SÃO PAULO

INSTITUTO DE FÍSICA DE SÃO CARLOS

ELICEO CORTES GOMEZ

Simulação da equação de Dirac em eletrodinâmica quântica de cavidades

SÃO CARLOS 

ELICEO CORTES GOMEZ

\title{
Simulação da equação de Dirac em eletrodinâmica quântica de cavidades
}

\author{
Dissertação apresentada ao Programa de Pós- \\ Graduação em Física do Instituto de Física de \\ São Carlos da Universidade de São Paulo, para \\ obtenção do título de Mestre em Ciências. \\ Área de concentração: Física Básica \\ Orientador: Prof. Dr. Miled Hassan Youssef \\ Moussa
}

\section{Versão Corrigida}

(Versão original disponível na Unidade que aloja o Programa)

São Carlos 


\section{AUTORIZO A REPRODUÇÃO E DIVULGAÇÃO TOTAL OU PARCIAL DESTE TRABALHO, POR QUALQUER MEIO CONVENCIONAL OU ELETRÔNICO PARA FINS DE ESTUDO E PESQUISA, DESDE QUE CITADA A FONTE.}

Ficha catalográfica elaborada pelo Serviço de Biblioteca e Informação do IFSC, com os dados fornecidos pelo(a) autor(a)

\section{Cortes Gomez, Eliceo}

Simulação da equação de Dirac em eletrodinâmica quântica de cavidades / Eliceo Cortes Gomez; orientador Miled Hassan Youssef Mousa - versão corrigida -- São Carlos, 2014

$$
62 \mathrm{p} \text {. }
$$

Dissertação (Mestrado - Programa de Pós-Graduação em Física Básica) -- Instituto de Física de São Carlos, Universidade de São Paulo, 2014.

1. Simulação quântica. 2. Eletrodinâmica quântica de cavidades. 3. Equação de Dirac. 4. O efeito

Zitterbewegung. I. Hassan Youssef Mousa, Miled, orient. II. Título. 





\section{AGRADECIMENTOS}

Primeiramente, agradeço a Deus por sua misericórdia e fidelidade ao longo desses dois anos do meu mestrado.

Aos meus pais Julio e Lourdes e aos meus irmãos que nunca deixaram de me apoiar mesmo nos instantes mais críticos de minha vida pessoal.

Aos meus amigos Edwin, Ruben, Oriana, Isaac, July, Manuel, Rogelio, Aldemar, Ricardo, Alfredo, Jeinny, Henrry, Andres, Emanuel, Martin por suas amizade e conversas inspiradoras.

Com relação aos professores e pesquisadores. Agradeço a meu orientador, Miled Hassan Youssef Moussa, pela orientação e por todo incentivo. Obrigado pela oportunidade de trabalhar em meu futuro.

Ao Wilson por sua amizade e sua participação direta neste trabalho.

Aos professores do IFSC e da UA que contribuíram em minha formação.

Também agradeço a Rafael e Víctor, pelas várias vezes que me ajudaram e por seu grande aporte neste trabalho.

Aos meus colegas de sala e de grupo Pedro, Cleverson e Gentil, pelas dicas certeiras.

Aos funcionários do IFSC-USP, pelo apoio administrativo e acadêmico.

À CAPES, pelo apoio financeiro. 

"O que prevemos raramente ocorre; o que menos esperamos geralmente acontece."

Benjamin Disraeli 



\section{Resumo}

GOMEZ, E.C. Simulação da equação de Dirac em eletrodinâmica quântica de cavidades. 2014. 62p. Dissertação (Mestrado em Ciências) - Instituto de Física de São Carlos, Universidade de São Paulo, São Carlos, 2015.

Neste trabalho apresentamos um protocolo para simular, no contexto da eletrodinâmica quântica de cavidades, a equação de Dirac $2+1$ D e $1+1$ D para uma partícula relativística livre, de spin 1/2. Especificamente, tratamos dois sistemas distintos: no primeiro consideramos um átomo de quatro níveis interagindo com dois modos da cavidade e quatro campos clássicos; no segundo, consideramos um átomo de três níveis interagindo com um modo da cavidade e dois campos clássicos. O primeiro sistema foi utilizado para simular a equação de Dirac $2+1$ D. Através do segundo sistema mostramos como simular a equação de Dirac $1+1$ D. Com esse sistema mostramos como manipular e controlar por meio das forças de acoplamentos dos campos, os valores da velocidade da luz e a energia de repouso da partícula relativística livre de Dirac simulada. Verificamos que a dinâmica de um elétron no formalismo da mecânica quântica relativística pode ser simulada usando experimentos em Eletrodinâmica Quântica de Cavidades. Neste contexto, analisamos o movimento oscilatório inesperado de uma partícula quântica relativística livre conhecido como Zitterbewegung.

Palavras-chave: Simulação quântica. Eletrodinâmica quântica de cavidades. Equação de Dirac. O efeito Zitterbewegung 



\begin{abstract}
GOMEZ, E.C. Simulation of Dirac equation in cavity quantum electrodynamics. 2014. 62p. Dissertação (Mestrado em Ciências) - Instituto de Física de São Carlos, Universidade de São Paulo, São Carlos, 2015.

In this work we present, in the context of cavity quantum electrodynamics, a protocol for simulating Dirac equation $2+1$ and $1+1$ for a relativistic free particle with spin $\frac{1}{2}$. Specifically, we deal with two different systems: In the first one we consider a four level atom interacting with two modes of the cavity and four classical fields; In the second system we deal consider a three level atom and interacting with one mode of the cavity and two classical fields. The first system was used to simulate a $2+1 \mathrm{D}$ Dirac equation. With the second system we show how to simulate a $1+1 \mathrm{D}$ Dirac equation. With these systems we show how to simulate and control through the field coupling strength, the values of the velocity of light and rest energy of the simulated Dirac's relativistic free particle. We verify that the dynamics of one electron in the formalism of relativistic quantum mechanics can be simulated using experiments in cavity quantum electrodynamics. In this context, we analyzed the unexpected but known oscillatory movement of a relativistic free quantum particle.
\end{abstract}

Keyword: Quantum simulation. Cavity quantum electrodynamics. Dirac equation. Zitterbewegung effect. 



\section{Lista de Figuras}

Figura 2.1 - Ilustração dos valores permitidos de energia de uma partícula relativística livre. . . . . . . . . . . . . . . . . . . . . . 30

Figura 3.1 - Representação esquemática de um sistema átomo-campo em EQC. Ilustra a interação de um átomo de dois níveis com um modo da cavidade com taxa de acoplamento $g$ e os processos de dissipação do sistema através das taxas $\kappa$ e $\gamma \ldots \ldots$. . . . . . . . . . . 34

Figura 4.1 - Configuração de níveis atômicos necessária para gerar o Hamiltoniano de Dirac 2+1 D. . . . . . . . . . . . . . . . . . 45

Figura 4.2 - Configuração de níveis atômicos, usada para gerar o Hamiltoniano de Dirac 1+1 D. . . . . . . . . . . . . . . . . . . . . 49

Figura 4.3 - Probabilidades obtidas a partir do Hamiltoniano efetivo (4.21) (curvas contínuas) e do Hamiltoniano sem aproximação (4.4) (curvas pontilhadas). (a) $P_{e}, P_{g}$ e $P$ e (b) $P_{0,0}, P_{1,1}$. . . . . . . . 52

Figura $4.4 \quad-\quad$ Probabilidades obtidas a partir do Hamiltoniano efetivo (3.39) (curvas contínuas) e do Hamiltoniano sem aproximação (3.29) (curvas pontilhadas). (a) $P_{e}, P_{g}$ e $P$ e (b) Populações do estado do campo $|0\rangle\left(P_{0}\right)$ e $|1\rangle\left(P_{1}\right)$. . . . . . . . . . . . . . 53

Figura 4.5 - Valor esperado, $\left\langle S_{1}\right\rangle$, para uma partícula relativística com diferentes massas. A curva linear azul representa um partícula sem massa movendo-se na velocidade da luz. As outras curvas representam partículas massivas com $\Lambda_{1}=3.11 R_{a}, \Lambda_{2}=0.99 R_{a}, \Lambda_{3}=0.57 R_{a}$ e $\Lambda_{4}=0.30 R_{a}$, respetivamente: . . . . . . . . . 56 



\section{Sumário}

1 Introdução

2 Equação de Dirac $\quad 23$

2.1 Introdução . . . . . . . . . . . . . . . . . . . . . . . . . . 23

2.2 Derivação da equação de Dirac $3+1 \mathrm{D} \quad \ldots \ldots \ldots \ldots$

$2.3 \quad$ Partícula livre de Dirac $\ldots \ldots \ldots \ldots$

$2.4 \quad$ O Efeito Zitterbewegung $\ldots \ldots \ldots \ldots$

3 Eletrodinâmica Quântica de Cavidades 33

3.1 Introdução . . . . . . . . . . . . . . . . . . . . . . . 33

3.2 Derivação do Hamiltoniano de Jaynes-Cummings ～. . . . . . . . . . . 35

3.3 Hamiltonianos Efetivos para interação dispersiva . . . . . . . . . . . . 39

4 Simulação da Equação de Dirac em EQC 43

4.1 Introdução . . . . . . . . . . . . . . . . . . . . 43

$4.2 \quad$ Hamiltoniano de Dirac via EQC . . . . . . . . . . . . . . . . 43

4.3 Validade das aproximações $\ldots \ldots \ldots \ldots$. . . . . . . . . 51

4.4 Simulação do Zitterbewegung via EQC . . . . . . . . . . . . . 54

5 Conclusão $\quad 57$

$\begin{array}{ll}\text { Referências } & 58\end{array}$ 



\section{Capítulo 1}

\section{Introdução}

No início do século passado, a Mecânica Quântica emergiu como consequência de uma série de descobrimentos e observações que deixaram em evidência, as dificuldades da física clássica para interpretar as propriedades do átomo, da radiação eletromagnética e de sua interação com a matéria, a nível dos seus constituintes fundamentais. (1-3) Desde então a mecânica quântica tem se mostrado, ao longo das décadas, como uma teoria extremamente bem sucedida. De tal forma que tornou-se uma ferramenta teórica básica para numerosas disciplinas de grande importância, como a Física Molecular, Atômica e Nuclear, a Física da Matéria Condensada, a Física de Partículas, etc.

Conjuntamente ao desenvolvimento da teoria da Mecânica Quântica, a descrição de fenômenos de altas energias requereu unificar as teorias da mecânica quântica com a relatividade restrita, fato que resultou em um novo campo da física que é a Mecânica Quântica Relativística. A primeira tentativa de unificar as idéias quânticas com a relatividade especial de Einstein, foi proposta em 1927 pelos físicos Oskar Klein e Walter Gordon. $(4,5)$ Mas essa teoria possuía uma problema ligado aos valores negativos para a densidade de probabilidade (fato que proíbe a interpretação probabilística da teoria), por que a equação conhecida como equação de Klein-Gordon é de segunda ordem no tempo. Devido a esta interpretação, em um primeiro momento, a equação de Klein-Gordon foi abandonada.

Em 1928 Paul Dirac resolveu o problema da densidade de probabilidade negativa propondo uma equação de onda relativística de primeira ordem, tanto nas variáveis temporais como espaciais, a qual descreve a dinâmica quântica dos férmions. (6) No ano de 1934 Pauli e V. F. Weisskopf solucionaram o problema da equação de Klein-Gordon interpretando a densidade de carga como densidade de probabilidade de corrente. (7) Isso solucionou o problema da equação de Klein-Gordon com relação aos valores negativos de densidade de probabilidade, mas, mesmo assim, a equação de Dirac possuía muitos proveitos quando comparada à equação de Klein-Gordon: já que a equação de Dirac fornece uma descrição natural do spin do elétron, prediz a existência da antimatéria (8) e reproduz precisamente o espectro do átomo de hidrogênio. Contudo, verificou-se que a equação de Dirac fez outras previsões interessantes, como o paradoxo de Klein (9) e o Zitterbewe- 
gung. $(10,11)$ O efeito do Zitterbewegung (ou "movimento trêmulo"), cujo nome foi dado por Schrödinger, se refere ao efeito observado quando a velocidade do eléctron não é uma constante do movimento na ausência de campos externos. Tal efeito tinha que ser de natureza quântica, já que não obedecia a primeira lei clássica do movimento de Newton. Schrödinger calculou a dependência do tempo da velocidade e da posição de uma partícula relativística livre, concluindo que, além de seu movimento clássico no tempo, a partícula tem um movimento oscilatório inesperado muito rápido com frequência, $\omega=2 E / \hbar$, que no mínimo tem um valor de $\omega_{\text {min }}=2 m_{0} c^{2} / \hbar \approx 10^{21} \mathrm{~Hz}$, e uma pequena amplitude da ordem do comprimento de onda de Compton, $\hbar / m_{0} c$. (12) Por esse motivo, o Zitterbewegung é experimentalmente inacessível. A partir de outra perspectiva, K. Huang (13) determinou as médias dos operadores de velocidade e posição e mostrou que o Zitterbewegung é devido à interferência dos estados correspondentes às energias negativas e positivas do elétron.

Por outro lado, nas últimas décadas, os avanços acelerados da Óptica Quântica possibilitaram aprofundar os conhecimentos estabelecidos acerca do campo de radiação, da matéria e da interação entre ambos, proporcionando resultados essenciais associados às propriedades da luz (14), e à manipulação de estados da matéria e de suas interações com campos de radiação. (15-18) Com relação à luz, os desenvolvimentos teóricos e experimentais no domínio das estatísticas de fotocontagens associadas aos estados comprimidos do campo de radiação, aprofundaram tanto o conhecimento do campo luminoso como a dicotomia entre suas descrições clássica e quântica. O antiagrupamento de fótons e a estatística sub-Poissoniana de fotocontagens constituem propriedades não-clássicas do campo luminoso, estabelecendo de forma inequívoca a necessidade da sua descrição quântica.

Dentro da Óptica Quântica, os avanços que aconteceram na eletrodinâmica quântica de cavidades (EQC), tanto no que se refere ao desenvolvimento teórico quanto às implementações experimentais, possibilitaram um amplo controle nas técnicas de manipulação da matéria e dos campos de radiação, sob o ponto de vista dos seus constituintes fundamentais. (19) Cavidades de alto fator de qualidade (alto-Q), fabricadas com material supercondutor, permitiram preparar fótons em estados bem definidos e acompanhar em tempo real a sua evolução. Nestas condições tão especiais, os átomos são conduzidos individualmente a interagir com apenas um modo da radiação, o que constitui um passo crucial para as observações experimentais de efeitos importantes, como a medida das oscilações de Rabi de átomos de Rydberg no vácuo e em campos coerentes fracos (20), a detecção do processo de decoerência de estados mesoscópicos de um modo da cavidade (21), a preparação de estados emaranhados massivos, envolvendo níveis de Rydberg de um par de átomos (22), e a implementação de portas lógicas quânticas. (23) Além do mais, foram demonstrados experimentalmente a geração e detecção de estados de Fock do campo de radiação. (24) Esse relativo domínio do mundo quântico rendeu o prêmio Nobel 
de Física de 2012 para a área de EQC. Esta maestria nos processos da EQC foi igualmente alcançada através de íons armadilhados, nos quais realizou-se a demostração das portas lógicas fundamentais, como a porta C-Not, a qual foi implementada via mapeamento dos dois bits quânticos de informação nos graus de liberdade interno e externo de um íon aprisionado. (25) Além da preparação de estados não-clássicos do campo vibracional (26), dentre os quais os estados emaranhados massivos (27), análogos aos que foram feitos na referência. (22)

Os resultados acima motivaram interessantes propostas nas simulações quânticas, pelas quais objetiva-se simular um sistema quântico de difícil acesso experimental através de outro com significativo grau de controle, ambos compartilhando, sob condições específicas, a mesma descrição matemática. Atualmente, o interesse na simulação quântica tem crescido rapidamente, devido a que um grande número de aplicações potenciais em Física, Química, Biologia, etc. Dentro os experimentos realizados usando simulação quântica, mencionamos a transição de fase quântica de um superfluido em um gás de átomos ultrafrios (28), a simulação da dinâmica de um spin com número quântico principal 1, 1/2 e 3/2 (29), e a evolução adiabática de um sistema de spin paramagnético a ferromagnético (30), usando as armadilhas iônicas, as quais são particularmente importantes para o propósito da simulação quântica. (31, 32)

Nos anos recentes, muita atenção tem sido dirigida à simulação quântica dos efeitos relativísticos. Lamata et al. (33), no contexto das armadilhas iônicas simularam a equação de Dirac 3+1 D (três dimensões espaciais e uma temporal) para uma partícula relativística livre de spin $1 / 2$, onde o bispinor de Dirac é representado por quatro estados internos iônicos, e a posição e o momento da partícula são associados às respectivas variáveis iônicas. Além, de propor uma forma de simular os efeitos relativísticos, como o Zitterbewegung e o paradoxo de Klein, sendo anos depois confirmados experimentalmente. $(34,35)$ Além das armadilhas iônicas, diferentes sistemas físicos foram utilizados para a proposição de protocolos para a simulação do zitterbewegung, como dispositivos semicondutores (12), cristais fotônicos (36) , átomos frios (37), condensados de Bose-Einstein $(38,39)$ e em férmions massivos na física do estado sólido. (40)

Motivados pelo atual domínio experimental, neste trabalho apresentamos um protocolo para simular, no contexto da EQC, o Hamiltoniano de Dirac 2+1 D e $1+1$ D para uma partícula relativística livre. Além do mais, mostramos teoricamente o comportamento semelhante ao Zitterbewegung no caso de uma dimensão, através da partícula armadilhada na cavidade de alto Q, usando a informação das quadraturas do campo eletromagnético e das forças de acoplamento associadas ao sistema átomo-campo.

Para alcançar e compreender questões relacionadas ao objetivo proposto, no capítulo 2 apresentaremos os conceitos da quântica relativística envolvidos neste trabalho, tais como a equação de Dirac e o Zitterbewegung. Já no capítulo 3 realizaremos uma breve revisão da EQC e introduziremos o formalismo do Hamiltoniano efetivo, o qual é 
uma ferramenta importante para nosso sistema. No capítulo 4, encontra-se a descrição da engenharia dos níveis atômicos e a técnica para a resolução do problema proposto. Finalmente no capítulo 5 apresentamos as conclusões gerais. 


\section{Equação de Dirac}

\subsection{Introdução}

A equação de Dirac é uma das pedras angulares da física contemporânea. Ela descreve de maneira correta a dinâmica dos férmions, os quais são os componentes fundamentais de nosso universo, prevê efeitos relativísticos interessantes (Zitterbewegung) e dar origem ao moderno paradigma da existência da antimatéria. Esses sucessos revolucionaram dramáticamente a forma de conceber a matéria e energia.

Neste capitulo apresentamos um estudo da equação de Dirac e suas soluções em $3+1$ D, com o objetivo de realizar comparações com os resultados obtidos no Capitulo 4. Em seguida, analisamos o conteúdo físico da equação Dirac para uma partícula livre relativística através do Zitterbewegung.

\subsection{Derivação da equação de Dirac $3+1 \mathrm{D}$}

Para o estudo de fenômenos de altas energias, precisamos de uma equação de onda relativística. Portanto, para ganharmos um pouco de intuição na descrição da equação de Dirac, vamos fazer uma analogia com a equação de onda da mecânica quântica não relativística, ou seja, a equação de Schrödinger. Neste contexto, sabemos que $i \hbar \frac{\partial}{\partial t}$ e $-i \hbar \vec{\nabla}$ são os operadores de energia e momento, respectivamente. Por conseguinte, se considerarmos o operador da energia relativística na ausência de potenciais, temos que:

$$
\hat{E}^{2}=c^{2} \hat{p}^{2}+m^{2} c^{4}
$$

ou:

$$
-\hbar^{2} \frac{\partial^{2}}{\partial t^{2}}=-c^{2} \hbar^{2} \nabla^{2}+m^{2} c^{4}
$$

onde $c$ é a velocidade da luz, $m$ e $\hat{p}$ são a massa da partícula e o operador momento , respectivamente. Assim, a equação de onda relativística para uma partícula livre, fica da seguinte forma: 


$$
\left[\frac{1}{c^{2}} \frac{\partial^{2}}{\partial t^{2}}-\nabla^{2}+\frac{m^{2} c^{2}}{\hbar^{2}}\right] \Psi(\vec{x}, t)=0
$$

A relação acima é conhecida como a equação de Klein-Gordon para partículas livres (41), que possui soluções da forma:

$$
\Psi(\vec{x}, t)=\exp \left[\frac{i}{\hbar}(\vec{p} \cdot \vec{x}-E t)\right]
$$

Se substituímos a Eq.(2.4) na equação de Klein-Gordon, obtemos duas soluções para a energia da partícula relativística, $E= \pm c \sqrt{|\vec{p}|^{2}+m^{2} c^{2}}$. Isso leva a um problema muito fundamental na equação de continuidade de Klein-Gordon, já que, a densidade de probabilidade pode ser positiva ou negativa.

Com a finalidade de mostrar este fato, tomamos o complexo conjugado, termo a termo, da Eq. (2.3) obtendo:

$$
\left[\frac{1}{c^{2}} \frac{\partial^{2}}{\partial t^{2}}-\nabla^{2}+\frac{m^{2} c^{2}}{\hbar^{2}}\right] \Psi^{*}(\vec{x}, t)=0
$$

Em seguida, multiplicamos a Eq.(2.3) à esquerda por $\Psi^{*}$ e a Eq.(2.5) à esquerda por $\Psi$ e subtraindo uma da outra obtemos

$$
\begin{gathered}
\vec{J}(\vec{x}, t)=\frac{i \hbar}{2 m_{0}}\left(\Psi^{*} \vec{\nabla} \Psi-\Psi \vec{\nabla} \Psi^{*}\right) \\
\rho(\vec{x}, t)=\frac{i \hbar}{2 m_{0} c^{2}}\left(\Psi^{*} \frac{\partial \Psi}{\partial t}-\Psi \frac{\partial \Psi^{*}}{\partial t}\right),
\end{gathered}
$$

em que, $\vec{J}(\vec{x}, t)$ é a densidade de corrente de probabilidade, a qual tem a mesma forma que o caso não relativístico. Portanto, o problema apresentado nesta teoria está na densidade de probabilidade, $\rho(\vec{x}, t)$. Isto pode ser visto claramente inserindo a Eq.(2.4) na Eq.(2.7), obtendo assim:

$$
\rho(\vec{x}, t)= \pm \frac{\sqrt{c^{2}|\vec{p}|^{2}+m^{2} c^{4}}}{m c^{2}} .
$$

Consequentemente, se perde a possibilidade de associar $\rho(\vec{x}, t)$ com uma densidade de probabilidade positiva. Devido a essa interpretação, a equação de Klein-Gordon não foi aceita para descrever o comportamento do elétron. Atualmente a compreensão das energias negativas é possível recorrendo à teoria de antipartículas.

Para o problema da densidade de probabilidade negativa, suspeita-se que a fonte da dificuldade atribuía-se ao fato de que a equação de Klein-Gordon é de segunda ordem no tempo. (42) Para solucionar esse inconveniente, Paul Dirac no ano 1928, usando a relação de energia relativística, propõe uma equação linear com relação à energia: 


$$
c^{2} p^{2}+m c^{4}=\left(c \vec{\alpha} \cdot \vec{p}+\beta m c^{2}\right)^{2},
$$

onde, $\vec{\alpha}=\left(\alpha_{1}, \alpha_{2}, \alpha_{3}\right)$ e $\beta$ são matrizes, tais que satisfazem as seguientes condições:

$$
\begin{gathered}
\left\{\alpha_{k}, \beta\right\}=0, \\
\alpha_{k}^{2}=\beta^{2}=I, \\
\left\{\alpha_{k}, \alpha_{l}\right\}=2 \delta_{k l} .
\end{gathered}
$$

Sendo, $k, l=1,2,3$. Por conseguinte, o problema se reduz a um problema puramente algébrico, onde temos que descobrir a forma das matrizes $\alpha_{k}$ e $\beta$. Se as matrizes $\alpha_{k}$ e $\beta$ são hermitianas, e da Eq.(2.11) sabemos que:

$$
\alpha_{k}^{2}=\left(\begin{array}{cccc}
1 & 0 & \cdots & 0 \\
0 & 1 & \cdots & 0 \\
\vdots & \vdots & \ddots & \vdots \\
0 & 0 & 0 & 1
\end{array}\right)=\left(\begin{array}{cccc}
a_{1}^{2} & 0 & \cdots & 0 \\
0 & a_{2}^{2} & \cdots & 0 \\
\vdots & \vdots & \ddots & \vdots \\
0 & 0 & 0 & a_{N}^{2}
\end{array}\right)
$$

Portanto, as matrizes $\alpha_{k}$ tem $N$ autovalores +1 e $N$ autovalores -1 , já que, $a_{m}= \pm 1$ onde $m=1,2,3, \ldots, N$. Além do mais, se calculamos o traço de $\alpha_{k}$ temos:

$$
\operatorname{tr}\left(\alpha_{k}\right)=\operatorname{tr}\left(\beta^{2} \alpha_{k}\right)=\operatorname{tr}\left(\beta \alpha_{k} \beta\right)
$$

onde usamos o fato que $\beta^{2}=I$, agora da Eq.(2.10) sabemos que $\alpha_{k}=-\beta \alpha_{k} \beta$, então da relação (2.14) obtemos que $\operatorname{tr}\left(\alpha_{k}\right)=0$. Consequentemente como o traço é a soma dos autovalores, os números de autovalores positivos e negativos têm que ser iguais. Então, a dimensão das matrizes $\alpha_{k}$ e $\beta$ é par, ou seja, $2 N$. As matrizes pares de mais baixa dimensão que satisfazem as condições $(2.10),(2.11)$ e $(2.12)$ são as matrizes $4 \times 4$. O conjunto de matrizes geralmente usado é:

$$
\begin{gathered}
\alpha_{k}=\left(\begin{array}{cc}
0 & \sigma_{k} \\
\sigma_{k} & 0
\end{array}\right) \\
\beta=\left(\begin{array}{cc}
I_{2 \times 2} & 0 \\
0 & -I_{2 \times 2}
\end{array}\right),
\end{gathered}
$$

sendo, $\sigma_{k}$, as matrizes de Pauli. 


$$
\sigma_{1}=\left(\begin{array}{cc}
0 & 1 \\
1 & 0
\end{array}\right), \quad \sigma_{2}=\left(\begin{array}{cc}
0 & -i \\
i & 0
\end{array}\right), \quad \sigma_{3}=\left(\begin{array}{cc}
1 & 0 \\
0 & -1
\end{array}\right)
$$

Finalmente, usando a Eq.(2.9) definimos o Hamiltoniano de Dirac,

$$
H_{D} \equiv c \vec{\alpha} \cdot \vec{p}+\beta m c^{2}
$$

obtendo, assim, a equação de onda relativística para partículas com spin $\frac{1}{2}$, conhecida como a equação de Dirac $3+1$ :

$$
H_{D} \psi=i \hbar \frac{\partial \psi}{\partial t}
$$

onde:

$$
\psi=\left(\begin{array}{l}
\psi_{1} \\
\psi_{2} \\
\psi_{3} \\
\psi_{4}
\end{array}\right)
$$

Este é o chamado espinor de Dirac. Através da equação de Dirac obtém-se a equação de continuidade, na qual a densidade de probabilidade, $\rho=\psi^{\dagger} \psi$, é sempre positiva. Portanto, Dirac resolve o problema da densidade de probabilidade negativa que se apresentava na equação de Klein-Gordon.

\subsection{Partícula livre de Dirac}

Para estudar o conteúdo físico da equação Dirac, vamos considerar primeiro o caso de uma partícula livre parada, $(\vec{p}=0)$. Neste contexto a equação Dirac se expressa da seguinte forma:

$$
i \hbar \frac{\partial \psi}{\partial t}=m c^{2} \beta \psi
$$

Além do mais, a equação de Dirac admite uma solução do tipo:

$$
\psi \sim u(\vec{p}) \exp [i(\vec{x} \cdot \vec{p} / \hbar)-i(E t / \hbar)]
$$

com:

$$
E= \pm c \sqrt{|\vec{p}|^{2}+m^{2} c^{2}}
$$

e , $u(\vec{p})$, um espinor de 4 componentes. Logo, a equação que devemos resolver, é:

$$
i \hbar \frac{\partial}{\partial t}\left\{u(0) \exp \left[\mp i\left(m c^{2} t / \hbar\right)\right]\right\}=m_{0} c^{2} \beta\left\{u(0) \exp \left[\mp i\left(m c^{2} t / \hbar\right)\right]\right\}
$$


onde substituímos as Eq.(2.22) e Eq.(2.23) na Eq.(2.21) com, $\vec{p}=0$. Tomamos primeiramente da Eq.(2.24) a dependência do tempo $\exp \left[-i m c^{2} t / \hbar\right]$, obtendo:

$$
m c^{2}\left(\begin{array}{c}
u_{A}(0) \\
u_{B}(0)
\end{array}\right)=m c^{2}\left(\begin{array}{cc}
I_{2 \times 2} & 0 \\
0 & -I_{2 \times 2}
\end{array}\right)\left(\begin{array}{c}
u_{A}(0) \\
u_{B}(0)
\end{array}\right) .
$$

Portanto, a Eq.(2.25) tem solução somente se as duas componentes inferiores do espinor $u(0)$ forem nulas, ou seja, $u_{B}(0)=0$. Agora, se tomarmos da Eq.(2.24) a dependência do tempo $\exp \left[+i m c^{2} t / \hbar\right]$ obtemos:

$$
-m c^{2}\left(\begin{array}{c}
u_{A}(0) \\
u_{B}(0)
\end{array}\right)=m c^{2}\left(\begin{array}{cc}
I_{2 \times 2} & 0 \\
0 & -I_{2 \times 2}
\end{array}\right)\left(\begin{array}{c}
u_{A}(0) \\
u_{B}(0)
\end{array}\right)
$$

então, a Eq. (2.26) tem solução quando as duas componentes superiores de $u(0)$ são nulas, isto é $u_{A}(0)=0$. Na teoria de Pauli, os spinores de dois componentes não nulos podem ter aspecto análogo a $\left(\begin{array}{l}1 \\ 0\end{array}\right)$ e $\left(\begin{array}{l}0 \\ 1\end{array}\right)$. Em vista disso, podemos obter 4 soluções linearmente independentes, a saber:

$$
\begin{aligned}
& \left(\begin{array}{l}
1 \\
0 \\
0 \\
0
\end{array}\right) \exp \left[-i m c^{2} t / \hbar\right], \quad\left(\begin{array}{l}
0 \\
1 \\
0 \\
0
\end{array}\right) \exp \left[-i m c^{2} t / \hbar\right] \\
& \left(\begin{array}{l}
0 \\
0 \\
1 \\
0
\end{array}\right) \exp \left[+i m c^{2} t / \hbar\right], \quad\left(\begin{array}{l}
0 \\
0 \\
0 \\
1
\end{array}\right) \exp \left[+i m c^{2} t / \hbar\right] .
\end{aligned}
$$

Concluímos que, as duas relações da Eq.(2.27) são soluções de energias positivas associadas a férmions, e as relações da Eq.(2.28) são soluções de energias negativas, as quais podem ser relacionadas com antiférmions . Na referência (7), mostra-se que a as soluções de energias negativas estão intimamente relacionadas ao fato de que a teoria de Dirac pode acomodar um pósitron, o qual é a antipartícula do elétron.

Vamos agora considerar o problema da partícula livre quando está em movimento $(\vec{p} \neq 0)$. Neste caso substituímos a Eq.(2.22) na Eq.(2.19) e obtemos

$$
\left(c \vec{\alpha} \cdot \vec{p}+\beta m c^{2}\right) u(\vec{p})=E u(\vec{p}),
$$

ou: 


$$
c\left(\begin{array}{cc}
0 & \vec{\sigma} \\
\vec{\sigma} & 0
\end{array}\right) \cdot \vec{p}\left(\begin{array}{l}
u_{A}(\vec{p}) \\
u_{B}(\vec{p})
\end{array}\right)+m c^{2}\left(\begin{array}{cc}
I_{2 \times 2} & 0 \\
0 & -I_{2 \times 2}
\end{array}\right)\left(\begin{array}{l}
u_{A}(\vec{p}) \\
u_{B}(\vec{p})
\end{array}\right)=E\left(\begin{array}{l}
u_{A}(\vec{p}) \\
u_{B}(\vec{p})
\end{array}\right)
$$

logo da $\operatorname{Eq}(2.29)$ podemos obter as seguintes relações:

$$
\begin{aligned}
& u_{A}(\vec{p})=\frac{c}{E-m c^{2}}(\vec{\sigma} \cdot \vec{p}) u_{B}(\vec{p}), \\
& u_{B}(\vec{p})=\frac{c}{E+m c^{2}}(\vec{\sigma} \cdot \vec{p}) u_{A}(\vec{p}),
\end{aligned}
$$

com;

$$
\vec{\sigma} \cdot \vec{p}=\sigma_{1} p_{1}+\sigma_{2} p_{2}+\sigma_{3} p_{3}=\left(\begin{array}{cc}
p_{3} & p_{1}-i p_{2} \\
p_{1}+i p_{2} & -p_{3}
\end{array}\right)
$$

Este sistema tem quatro soluções linearmente independentes, que podemos obter da seguinte forma: vamos considerar o caso quando $E=c \sqrt{|\vec{p}|^{2}+m^{2} c^{2}}>0$, além disso, usarmos os spinores de dois componentes $\left(\begin{array}{l}1 \\ 0\end{array}\right)$ e $\left(\begin{array}{l}0 \\ 1\end{array}\right)$, para $u_{A}(\vec{p})$. Portanto, levando em conta estas considerações na Eq.(2.31) temos:

$$
u_{B}^{(1)}(\vec{p})=\left(\begin{array}{c}
\frac{p_{3} c}{E+m c^{2}} \\
\frac{\left(p_{1}+i p_{2}\right) c}{E+m c^{2}}
\end{array}\right) \quad e \quad u_{B}^{(2)}(\vec{p})=\left(\begin{array}{c}
\frac{\left(p_{1}-i p_{2}\right) c}{E+m c^{2}} \\
-\frac{p_{3} c}{E+m c^{2}}
\end{array}\right)
$$

Para o caso quando $E=-c \sqrt{|\vec{p}|^{2}+m_{0}^{2} c^{2}}<0$, podemos, à semelhança do que fizemos para $E>0$, chegar a:

$$
u_{A}^{(1)}(\vec{p})=\left(\begin{array}{c}
\frac{-p_{3} c}{|E|+m c^{2}} \\
\frac{-\left(p_{1}+i p_{2}\right) c}{|E|+m c^{2}}
\end{array}\right) \quad u_{A}^{(2)}(\vec{p})=\left(\begin{array}{c}
\frac{-\left(p_{1}-i p_{2}\right) c}{|E|+m c^{2}} \\
\frac{p_{3} c}{|E|+m c^{2}}
\end{array}\right)
$$

Por conseguinte, obtemos quatro soluções independentes a saber:

$$
\begin{aligned}
& u^{(1)}(\vec{p})=N_{1}\left(\begin{array}{c}
1 \\
0 \\
u_{B}^{(1)}
\end{array}\right)=N_{1}\left(\begin{array}{c}
1 \\
0 \\
\frac{p_{3} c}{E+m c^{2}} \\
\frac{\left(p_{1}+i p_{2}\right) c}{|E|+m c^{2}}
\end{array}\right), \\
& u^{(2)}(\vec{p})=N_{1}\left(\begin{array}{c}
0 \\
1 \\
u_{B}^{(1)}
\end{array}\right)=N_{1}\left(\begin{array}{c}
0 \\
1 \\
\frac{\left(p_{1}-i p_{2}\right) c}{E+m c^{2}} \\
\frac{-p_{3} c}{E+m c^{2}}
\end{array}\right),
\end{aligned}
$$




$$
\begin{aligned}
& u^{(3)}(\vec{p})=N_{1}\left(\begin{array}{c}
u_{A}^{(1)} \\
1 \\
0
\end{array}\right)=N_{1}\left(\begin{array}{c}
\frac{-p_{3} c}{|E|+m c^{2}} \\
\frac{-\left(p_{1}+i p p_{2}\right) c}{|E|+m c^{2}} \\
1 \\
0
\end{array}\right), \\
& u^{(4)}(\vec{p})=N_{1}\left(\begin{array}{c}
u_{A}^{(2)} \\
0 \\
1
\end{array}\right)=N_{1}\left(\begin{array}{c}
\frac{-\left(p_{1}-i p_{2}\right) c}{|E|+m c^{2}} \\
\frac{p_{3} c}{|E|+m c^{2}} \\
0 \\
1
\end{array}\right) .
\end{aligned}
$$

Onde a constante de normalização, $N_{1}$, será determinada a seguir. As duas soluções para cada caso representam as duas direções que pode ter o spin de cada partícula. Ou seja, para $E>0$ temos as duas soluções $u^{(1)}(\vec{p})$ e $u^{(2)}(\vec{p})$, que descrevem as duas possíveis orientações do spin do elétron, e para $E<0$ temos as duas soluções $u^{(3)}(\vec{p})$ e $u^{(4)}(\vec{p})$, as quais representam as duas possíveis orientações do spin do pósitron. Para finalizar, normalizamos a função de onda $u(\vec{p})$, impondo que :

$$
u^{(r) \dagger}(\vec{p}) u^{(r)}(\vec{p})=\frac{|E|}{m c^{2}},
$$

com $r=1,2,3,4$. Portanto, $N_{1}$ é determinado da seguinte forma:

$$
u^{(1) \dagger}(\vec{p}) u^{(1)}(\vec{p})=N_{1}^{2}\left(\begin{array}{cccc}
1 & 0 & \frac{p_{3} c}{E+m c^{2}} & \frac{\left(p_{1}-i p_{2}\right) c}{|E|+m c^{2}}
\end{array}\right)\left(\begin{array}{c}
1 \\
0 \\
\frac{p_{3} c}{E+m c^{2}} \\
\frac{\left(p_{1}+i p_{2}\right) c}{|E|+m c^{2}}
\end{array}\right)=\frac{|E|}{m c^{2}}
$$

então:

$$
N_{1}^{2}\left(1+\frac{p^{2} c^{2}}{\left(|E|+m c^{2}\right)^{2}}\right)=N_{1}^{2} \frac{2|E|}{|E|+m c^{2}}=\frac{|E|}{m c^{2}}
$$

portanto:

$$
N_{1}=\sqrt{\frac{|E|+m c^{2}}{2 m c^{2}}} .
$$

Agora, para normalizar a Eq.(2.22), vamos usar a Eq.(2.38) obtendo:

$$
\int \psi^{\dagger} \psi d^{3} \vec{r}=N_{2}^{2} \int u^{(r) \dagger}(\vec{p}) u^{(r)}(\vec{p}) d^{3} \vec{r}=1
$$

assim:

$$
N_{2}=\sqrt{m c^{2} /|E| V},
$$

onde $V$ é o volume de um cubo de lado $L$ no espaço. 
Figura 2.1 - Ilustração dos valores permitidos de energia de uma partícula relativística livre.

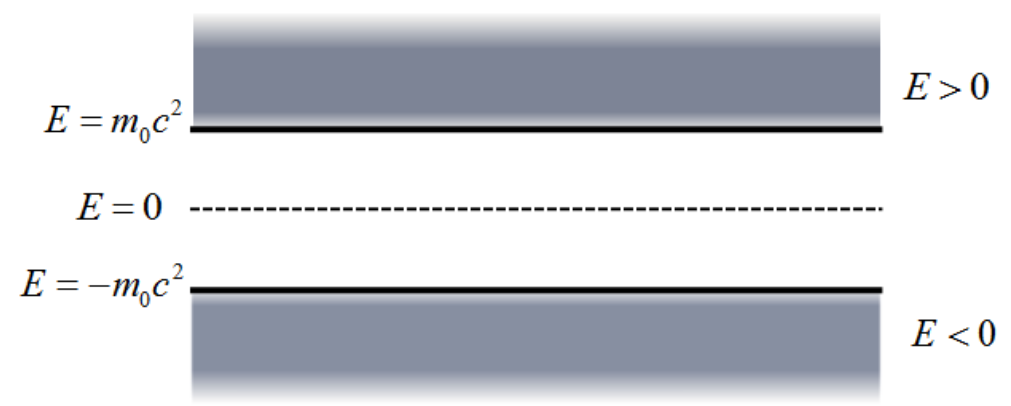

Fonte: Elaborada pelo autor.

Finalmente as soluções de onda plana normalizadas para um dado $\vec{p}$ são:

$$
\begin{gathered}
\psi_{+}=\sqrt{\frac{m c^{2}}{|E| V}} u^{(1 \text { ou } 2)}(\vec{p}) \exp [i(\vec{x} \cdot \vec{p} / \hbar)-i(E t / \hbar)], \\
\psi_{-}=\sqrt{\frac{m c^{2}}{|E| V}} u^{(3 \text { ou } 4)}(\vec{p}) \exp [i(\vec{x} \cdot \vec{p} / \hbar)+i(|E| t / \hbar)],
\end{gathered}
$$

sendo $\psi_{+}$as soluções para energias positivas, e $\psi_{-}$para as energias negativas. Quando $V \rightarrow \infty$, os valores permitidos formam um contínuo para soluções de energias positivas $m c^{2} \leq E<\infty$ e para energias negativas $-\infty<E \leq-m c^{2}$, ver Fig. (2.1).

As soluções $\psi$ - para a equação de Dirac são interpretadas usando a teoria de buracos. (41) Neste contexto, se um elétron do mar de Dirac (mar infinito de elétrons com energias negativas), ou seja, um elétron com $E<0$ absorve um fóton com energia maior que a energia do gap, $2 m c^{2}$, cria-se um estado buraco no mar de Dirac. O buraco é interpretado na teoria de "buracos" como o pósitron. Portanto, através da extensão da teoria de Dirac de partícula única para a teoria de buraco se prediz o pósitron como antipartícula do elétron, o que foi observado depois, experimentalmente. (43) Além disto, as duas soluções de energias positivas e negativas, interferem entre si gerando o efeito Zitterbewegung, que será melhor explicado em mais detalhes em seguida.

\subsection{O Efeito Zitterbewegung}

Nesta seção estudaremos o movimento de um elétron livre na representação de Heisenberg conforme a teoria de Dirac e analisaremos as equações do movimento de Heisenberg. Para iniciar este estudo, vamos escrever na representação de Heisenberg a evolução 
temporal do operador posição da partícula relativística livre da seguinte maneira:

$$
\dot{S}_{k}(t)=\frac{i}{\hbar}\left[H_{D}, S_{k}\right]=\frac{i}{\hbar}\left[c \alpha_{j} p_{j}+\beta m c^{2}, S_{k}\right]
$$

onde, $k, j=1,2,3$. Da Eq.(2.44) obtemos imediatamente:

$$
\dot{S}_{k}(t)=\frac{i c}{\hbar}\left[\alpha_{j} p_{j}, S_{k}\right]=c \alpha_{k}(t)
$$

Como os autovalores de $\alpha_{k}$ são \pm 1 , posto que, $\alpha_{k}^{2}=I$. Então, o operador velocidade da Eq.(2.45) possui autovalores $\pm c$. Por outro lado, sabemos que os elétrons tem velocidades muito inferiores à velocidade da luz, o que aparentemente é incoerente com relação ao resultado da Eq.(2.45). No entanto, a contradição não é real já que a velocidade teórica do resultado anterior é num instante dado, enquanto as velocidades observadas são sempre médias da velocidade durante intervalos de tempo consideráveis. (44) Com isso, o próximo passo é verificar como muda a velocidade do elétron com o tempo. Para isto, vamos estudar o operador $\alpha_{k}$ na representação de Heisenberg:

$$
\dot{\alpha}_{k}(t)=\frac{i}{\hbar}\left[H_{D}, \alpha_{k}\right]
$$

agora, usando as relações $(2.11),(2.12)$ e $\left\{H_{D}, \alpha_{k}\right\}=2 c p_{k}$ na Eq.(2.46) obtemos

$$
\dot{\alpha}_{k}(t)=\frac{i}{\hbar}\left(-2 \alpha_{k} H_{D}+2 c p_{k}\right)
$$

como $p_{k}$ e $H_{D}$ são grandezas conservadas, então derivando a Eq.(2.47) e integrando o resultado deduzimos que:

$$
\dot{\alpha}_{k}(t)=\dot{\alpha}_{k}^{0} e^{-2 i H_{D} t / \hbar},
$$

onde impomos a condição inicial $\dot{\alpha}_{k}(t=0)=\dot{\alpha}_{k}^{0}$. Logo, substituindo a Eq.(2.48) na Eq.(2.47), obtemos:

$$
\alpha_{k}(t)=\frac{i \hbar}{2} \dot{\alpha}_{k}^{0} e^{-2 i H_{D} t / \hbar} H_{D}^{-1}+c p_{k} H_{D}^{-1}
$$

ou:

$$
\alpha_{k}(t)=c p_{k} H_{D}^{-1}+\left(\alpha_{k}(0)-c p_{k} H_{D}^{-1}\right) e^{-2 i H_{D} t / \hbar} .
$$

Agora, usando a Eq.(2.50) em Eq.(2.45) podemos obter o operador velocidade da partícula relativística:

$$
\dot{S}_{k}(t)=c^{2} p_{k} H_{D}^{-1}+c\left(\alpha_{k}(0)-c p_{k} H_{D}^{-1}\right) e^{-2 i H_{D} t / \hbar}
$$

e se integramos em $t$ temos:

$$
S_{k}(t)=S_{k}(0)+c^{2} p_{k} H_{D}^{-1} t+\frac{i \hbar c}{2}\left(\alpha_{k}(0)-c p_{k} H_{D}^{-1}\right) H_{D}^{-1}\left[e^{-2 i H_{D} t / \hbar}-1\right]
$$


Os dois primeiros termos da Eq.(2.52) descrevem o movimento de uma partícula clássica livre. O último termo é característico da mecânica quântica relativística, o qual é responsável pela adição de um movimento oscilatório com uma alta frequência, $\omega=2 E / \hbar$, que no mínimo tem um valor de $\omega_{\text {min }}=2 m c^{2} / \hbar \approx 10^{21} \mathrm{~Hz}$, e uma pequena amplitude da ordem, $h / m c$, do comprimento de onda de Compton. Este movimento oscilatório é o chamado Zitterbewegung, conforme batizado por Schrödinger. (11)

As relações não convencionais (2.45) e (2.50) surgem devido à existência de soluções de energias negativas. Para mostrar esse fato, vamos usar a representação de Schrödinger. A função de onda geral de uma partícula livre relativística é:

$$
\begin{aligned}
\psi(\vec{S}, t)= & \sum_{\vec{p}} \sum_{r=1,2} \sqrt{\frac{m c^{2}}{|E| V}} c_{\vec{p}, r} u^{(r)}(\vec{p}) \exp \left[\frac{i \vec{p} \cdot \vec{S}}{\hbar}-\frac{i|E| t}{\hbar}\right] \\
& +\sum_{\vec{p}} \sum_{r=3,4} \sqrt{\frac{m c^{2}}{|E| V}} c_{\vec{p}, r} u^{(r)}(\vec{p}) \exp \left[\frac{i \vec{p} \cdot \vec{S}}{\hbar}+\frac{i|E| t}{\hbar}\right],
\end{aligned}
$$

onde $c_{p, r}$ é a amplitude da onda. (7) Logo, para analisar nesta representação a velocidade física da partícula relativística calculamos:

$$
\begin{aligned}
\left\langle\alpha_{k}\right\rangle= & \int_{v} \psi^{\dagger}(\vec{S}, t) \alpha_{k} \psi(\vec{S}, t) d^{3} x \\
= & \sum_{\vec{p}} \sum_{r=1,2}\left|c_{\vec{p}, r}\right|^{2} \frac{p_{k} c}{|E|}-\sum_{\vec{p}} \sum_{r=3,4}\left|c_{\vec{p}, r}\right|^{2} \frac{p_{k} c}{|E|} \\
& +\sum_{\vec{p}} \sum_{r=1,2} \sum_{r^{\prime}=3,4} \frac{m c^{2}}{|E|}\left[c_{\vec{p}, r^{\prime}}^{\star} c_{\vec{p}, r} u^{(r \prime) \dagger} \alpha_{k} u^{(r)} \exp (-2 i|E| t / \hbar)\right. \\
& \left.+c_{\vec{p}, r^{\prime}} c_{\vec{p}, r}^{\star} u^{(r) \dagger} \alpha_{k} u^{\left(r^{\prime \prime}\right)} \exp (2 i|E| t / \hbar)\right],
\end{aligned}
$$

onde usamos:

$$
\int d^{3} \vec{S} \exp \left[\vec{S} \cdot\left(\vec{p}-\vec{p}^{\prime}\right) / \hbar\right]=V \delta_{\vec{p} p^{\prime}}
$$

e:

$$
u^{(r) \dagger} \alpha_{k} u^{(r)}=\frac{p_{k}}{m c} .
$$

A Eq.(2.54) mostra que o zitterbewegung está completamente ausente para um pacote de onda que compõe-se exclusivamente de soluções de energias positivas (negativas). Portanto, o zitterbewegung é possível somente quando há sobreposição das soluções de energias positiva e negativa. 
Capítulo 3

\section{Eletrodinâmica Quântica de Cavidades}

\subsection{Introdução}

O estudo da interação radiação com a matéria mudou definitivamente quando Dirac e Fermi. (45, 46), associaram cada modo do campo da radiação com um oscilador harmônico quantizado, sendo isto fundamental para a teoria quântica da radiação. A quantização do campo de radiação forneceu a explicação do fenômenos como emissão espontânea (47), efeito fotoelétrico (48), o deslocamento de Lamb (49), entre outros. Consequentemente, os estudos da interação átomo-campo iniciam-se neste novo contexto, onde utiliza-se o campo e o átomo quantizados, diferentemente da teoria semiclássica de interação átomocampo, onde o campo é tratado classicamente e o átomo quanticamente.

O resultado deste novo sistema quântico da origem à eletrodinâmica quântica de cavidades (EQC), a qual pode ser definida, em poucas palavras, como a física da interação entre um spin e um oscilador. (19) Ou seja, no domínio da EQC pode-se entender um dos sistemas quânticos mais simples e ao mesmo tempo não trivial, o qual consiste em um átomo de dois níveis (formalmente análogo a um sistema de spin 1/2), e um campo electromagnético quantizado como um conjunto de osciladores harmônicos, interagindo dentro de uma cavidade; sendo um dos osciladores ressonante ou aproximadamente ressonante com a transição atômica, de tal maneira que os outros osciladores sejam negligenciados. Este sistema foi usado por Jaynes-Cummings para descrever a interação radiação-materia quanticamente. (50) Em uma primeira abordagem o modelo de Jaynes-Cummings foi considerado simplesmente como um experimento mental, não obstante, com os avanços nas técnicas de preparação e manipulação de átomos individuais, tornou-se possível a comprovação experimental de tal modelo.

Nos experimentos típicos em EQC a cavidade usada consiste num interferômetro de Fabry - Perot construído de Nióbio no estado supercondutor, com um alto fator de qualidade de $Q=\omega / \kappa \sim 10^{9}$, onde $\kappa$ é a taxa de decaimento do fótons da cavidade e $\omega$ é a frequência do modo armadilhado dentro da cavidade. Este fator de qualidade implica que os campos de radiação com frequência na região de microondas, tempo de vida da ordem de milissegundo. O que possibilita realizar os experimentos em EQC com significativa 
Figura 3.1 - Representação esquemática de um sistema átomo-campo em EQC. Ilustra a interação de um átomo de dois níveis com um modo da cavidade com taxa de acoplamento $g$ e os processos de dissipação do sistema através das taxas $\kappa$ e $\gamma$.

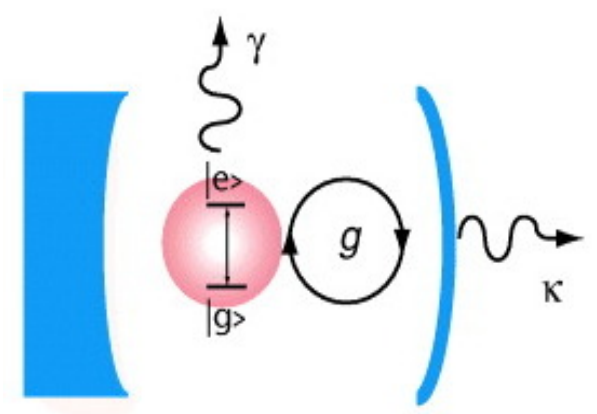

Fonte: FARAON, A (52)

fidelidade, já que, o tempo típico de interação átomo-campo é da ordem de $1 / g \sim 10^{-5} s$, sendo $g$ o parâmetro de acoplamento átomo-campo. Os processo dissipativos dos sistemas de EQC como a emissão espontânea e espelhos imperfeitos, são incluídos através da taxa de decaimento do átomo, $\gamma$, e $\kappa$. Esses parâmetros são ilustrados na figura 3.1.

Para cavidades com modos na região de microondas utilizam-se estados de Rydberg circulares, os quais consideram-se como átomos de dois níveis. Assim, o mecanismo do experimento baseia-se em um feixe de átomos de Rydberg preparado via zonas de Ramsey (51) antes de interagir com a cavidade que posteriormente penetra na cavidade, onde interage com o campo. No fim da qual há uma região, onde é aplicado um campo elétrico intenso que permite detectar de forma seletiva o estado atômico. Este sistema usado por Haroche e colaboradores (15), tem uma configuração flexível e versátil, de tal forma que as frequências do campo e das transições atômicas podem ser ajustadas para o estudo de interações ressonantes ou dispersivas entre o átomo e o campo de radiação na cavidade. As interações ressonantes apresentam-se quando a frequência atômica é igual à frequência do campo. Este tipo de interação entre átomo-campo é descrita pelo Hamiltoniano de Jaynescummings. As interações dispersivas, aparecem quando a frequência de transição atômica é muito diferente da frequência do campo, ou seja, que dentro da cavidade os átomos não podem nem emitir nem absorver fótons. Entretanto, o efeito da interação dispersiva entre o átomo e o campo é de deslocar os níveis de energia atômicos, originando o já conhecido efeito Stark.

Neste capítulo abordaremos especificamente a derivação do hamiltoniano de JaynesCummings e seu hamiltoniano efetivo para interações dispersivas, já que, as interações átomo-campo envolvidas neste trabalho são interações não ressonantes. 


\subsection{Derivação do Hamiltoniano de Jaynes-Cummings}

Nesta seção, vamos analisar a interação ressonante entre um modo da cavidade e o átomo, o qual foi primeiramente analisado em detalhe por Jaynes e Cummings em 1963. (50) O modelo de Jaynes-Cummings, descreve a interação de um átomo de dois níveis com um único modo do campo de radiação quantizado. O êxito deste modelo, mesmo sob uma descrição inteiramente quântica, é que possui solução exata e suas predições podem ser verificadas no laboratório.

Para iniciar a derivação do hamiltoniano de Jaynes-Cummings, partimos do hamiltoniano que descreve a interação do campo de radiação com o átomo:

$$
\mathcal{H}=\sum \frac{1}{2 m}\left[\vec{p}_{i}-\frac{e \vec{A}\left(\vec{r}_{i}, t\right)}{c}\right]^{2}+V\left(\vec{r}_{i}\right)+\mathcal{H}_{c},
$$

primeiramente vamos considerar os dois primeiros termos da equação em cima, onde $\vec{p}_{i}$ é o momento para i-ésimo elétron, $V\left(\vec{r}_{i}\right)$ o potencial de Coulomb , $\vec{A}\left(\vec{r}_{i}, t\right)$ é o potencial vetor, $m$ e $e$ são a massa e a carga do elétron e $c$ a velocidade da luz. Agora usando em $\mathcal{H}$ o gauge de Coulomb $\left(\vec{\nabla} \cdot \vec{A}\left(\vec{r}_{i}, t\right)=0\right)$ e o fato que $\vec{A}^{2}\left(\vec{r}_{i}, t\right) \ll 1$, obtemos:

$$
\mathcal{H}=\sum_{i}\left[\frac{\vec{p}_{i}^{2}}{2 m}+V\left(\vec{r}_{i}\right)\right]-\frac{e}{m c} \sum_{i} \vec{A}\left(\vec{r}_{i}, t\right) \cdot \vec{p}_{i}+\mathcal{H}_{c},
$$

o primeiro e o segundo termos da Eq.(3.1) descrevem o hamiltoniano do átomo, $\mathcal{H}_{\mathcal{A}}$, e o hamiltoniano de interação radiação-átomo, $\mathcal{H}_{I N T}$, respectivamente. O terceiro termo é o hamiltoniano do campo de radiação quantizado:

$$
\mathcal{H}_{c}=\sum_{\vec{k}, \beta} \hbar \omega_{\vec{k}, \beta}\left(a_{\vec{k}, \beta}^{\dagger} a_{\vec{k}, \beta}+1 / 2\right)
$$

o qual tem a forma do hamiltoniano do oscilador harmônico quântico, de frequência $\omega_{\vec{k}, \beta}$ e operador número $a_{\vec{k}, \beta}^{\dagger} a_{\vec{k}, \beta}$, sendo $\vec{k}$ o vetor de onda e $\beta$ nos diz que existem dois estados linearmente independientes de polarização a cada vetor de onda $\vec{k}$. Portanto, cada modo do $\mathcal{H}_{c}$ é dinamicamente equivalente a um oscilador harmônico quântico. (53)

Logo, devido a que desejamos analisar a interação átomo-campo no contexto da mecânica quântica, precisamos quantizar o campo de matéria e o termo de interação radiação-matéria dados na Eq.(3.1). Primeiramente, vamos quantizar $\mathcal{H}_{\mathcal{A}}$, então usando o formalismo da segunda quantização (54), definimos o hamiltoniano do átomo da seguinte forma:

$$
\mathcal{H}_{i}=\int d^{3} \vec{r} \Phi_{i}^{\dagger} \mathcal{H}_{\mathcal{A}} \Phi_{i}
$$

Sendo: 


$$
\begin{gathered}
\Phi_{i}=\sum_{j} b_{i j} \phi_{i j}, \\
\Phi_{i}^{\dagger}=\sum_{j} b_{i j}^{\dagger} \phi_{i j}^{\dagger},
\end{gathered}
$$

os operadores de campo do i-ésimo elétron, onde $b_{i j}\left(b_{i j}^{\dagger}\right)$ é o operador fermiônico de destruição (criação) do j-ésimo nível de energia e $\left\{\phi_{i j}\right\}$ constitui uma base que procede da solução da equação de Schrödinger. Aliás, a base $\left\{\phi_{i j}\right\}$ define um conjunto de autofunções ortonormais. Portanto, usando as relações (3.4) e (3.5) na Eq.(3.3), obtemos a quantização do hamiltoniano do átomo:

$$
H_{A}=\sum_{i} \mathcal{H}_{i}=\sum_{i} \int d^{3} \vec{r} \Phi_{i}^{\dagger}\left(\frac{\vec{p}_{i}^{2}}{2 m}+V\left(\vec{r}_{i}\right)\right) \Phi_{i}=\sum_{i j} E_{i j} b_{i j}^{\dagger} b_{i j}
$$

Onde $E_{i j}$ é a autoenergia da autofunção $\phi_{i j}$. Vamos agora obter a quantização do hamiltoniano de interação radiação-átomo. Para isto, consideraremos a interação da radiação com um único elétron de valência, ou seja, $i=1$ (usaremos esse fato de aqui em diante). Então, da teoria quântica da radiação (7), temos que o potencial vetor quantizado $\vec{A}(\vec{r}, t=0)$ é dado por:

$$
\vec{A}(\vec{r}, 0)=\sum_{\vec{k}, \beta} \frac{c}{\sqrt{V}} \sqrt{\frac{\hbar}{2 \omega_{\vec{k}}}}\left[a_{\vec{k}, \beta} \vec{\epsilon}^{(\beta)} e^{i \vec{k} \cdot \vec{r}}+a_{\vec{k}, \beta}^{\dagger} \vec{\epsilon}^{(\beta)} e^{-i \vec{k} \cdot \vec{r}}\right]
$$

sendo $\vec{\epsilon}$ o vetor de polarização, $V=L^{3}$ é o volume do cubo que contém o campo do potencial vetor. Vale a pena notar que o potencial vetor da Eq.(3.7) está expressado na representação do Schrödinger. Assim, usando a Eq.(3.3), mas para o caso do $\mathcal{H}_{I N T}$ temos:

$$
H_{I N T}=\int d^{3} \vec{r} \Phi^{\dagger} \mathcal{H}_{I N T} \Phi
$$

logo, utilizando as relações (3.4), (3.5) obtemos:

$$
H_{I N T}=\sum_{l j} b_{l}^{\dagger} b_{j} \int d^{3} \vec{r} \phi_{l}^{*}\left[-\frac{e}{m c} \vec{A}(\vec{r}, 0) \cdot \vec{p}\right] \phi_{j}
$$

Se substituímos a Eq.(3.7) na Eq.(3.9) temos:

$$
H_{I N T}=\hbar \sum_{l j} \sum_{\vec{k}, \beta} b_{l}^{\dagger} b_{j}\left(g_{l, j ; \vec{k}, \beta} a_{\vec{k}, \beta}+g_{l, j ; \vec{k}, \beta}^{*} a_{\vec{k}, \beta}^{\dagger}\right),
$$


onde $g_{l, j ; \vec{k}, \beta}$ é a frequência de Rabi definida da seguinte forma:

$$
g_{l, j ; \vec{k}, \beta}=-\frac{e}{m}\left(\frac{1}{2 \hbar \omega_{\vec{k}} V}\right)^{1 / 2} \int d^{3} \vec{r} \phi_{l}^{*} e^{i \vec{k} \cdot \vec{r}} \vec{\epsilon}^{(\beta)} \cdot \vec{p} \phi_{j}
$$

Por outro lado, se consideramos a aproximação de dipolo elétrico $\vec{k} \cdot \vec{r} \ll 1$, o qual implica que a variação espacial do potencial vetor é negligenciável no hamiltoniano de radiaçãoátomo. Consequentemente, pode-se substituir $\vec{A}(\vec{r}, 0)$ por $\vec{A}\left(\vec{r}_{0}, 0\right)$, onde $\vec{r}_{0}$ é um ponto fixo tomado no interior do sistema de cargas. Levando em conta as considerações anteriores, podemos escrever:

$$
g_{l, j ; \vec{k}, \beta}=-i\left(\frac{1}{2 \hbar \omega_{\vec{k}} V}\right)^{1 / 2} \omega_{l j} e^{i \vec{k} \cdot \vec{r}_{0}} \vec{\epsilon}^{(\beta)}\left\langle\phi_{l}|e \vec{r}| \phi_{j}\right\rangle
$$

com $\omega_{l j}=\left(E_{l}-E_{j}\right) / \hbar$. Quantizados os hamiltonianos $H_{A}$ e $H_{I N T}$, vamos agora a escrever o problema da interação do campo de radiação com o átomo na representação de interação, por meio do hamiltoniano de interação:

$$
H_{I}=e^{i H_{0} t / \hbar} H_{I N T} e^{-i H_{0} t / \hbar},
$$

onde $H_{0}=H_{A}+H_{C}$. Substituindo na Eq.(3.13) as Eqs. (3.2), (3.6) e (3.10) e usando a fórmula de Baker - Haussdorf obtemos:

$$
H_{I}=\hbar \sum_{l j} \sum_{\vec{k}, \beta} b_{l}^{\dagger} b_{j}\left(g_{l, j ; \vec{k}, \beta} a_{\vec{k}, \beta} e^{-i\left(\omega_{\vec{k}}-\omega_{l j}\right) t}+g_{l, j ; \vec{k}, \beta}^{*} a_{\vec{k}, \beta}^{\dagger} e^{i\left(\omega_{\vec{k}}+\omega_{l j}\right) t}\right) .
$$

Esta importante relação é útil para estudar de maneira particular um átomo de dois níveis com estado fundamental (1) e o exitado (2), e com frequência de transição $\omega_{0}=\omega_{21}$, interagindo com um único modo quantizado do campo eletromagnético de uma cavidade óptica, de frequência $\omega$. Neste contexto, da Eq.(3.14) obtemos o hamiltoniano:

$$
H_{I}=\hbar g\left[b_{2}^{\dagger} b_{1} a e^{-i\left(\omega-\omega_{0}\right) t}+b_{1}^{\dagger} b_{2} a^{\dagger} e^{i\left(\omega-\omega_{0}\right) t}\right]
$$

onde $g_{21}=g_{12}^{*}=g$ é real. Além disso, utilizamos a aproximação de onda girante ou RWA, do ingles "Rotating Wave Approximation", a qual consiste em dezprezar a contribuição dos termos muito oscilantes que em media não contribuem efetivamente para a dinâmica do sistema. (55) Portanto devemos notar que para garantir aproximação de onda girante impõe-se que $\sqrt{\bar{n}+1} g \ll \omega+\omega_{0}$, onde, $\bar{n}$ é o número médio de fótons na cavidade.

Finalmente se consideramos nas equações (3.15) e (3.6) a correspondência entre os operadores de pseudo-spin $\left(\sigma_{ \pm} \mathrm{e} \sigma_{z}\right)$ e os operadores fermiônicos a saber: 


$$
\begin{gathered}
\sigma_{+}=|2\rangle\langle 1| \longleftrightarrow b_{2}^{\dagger} b_{1}, \\
\sigma_{-}=|1\rangle\langle 2| \longleftrightarrow b_{1}^{\dagger} b_{2}, \\
\sigma_{z}=|2\rangle\langle 2|-| 1\rangle\langle 1| \longleftrightarrow b_{2}^{\dagger} b_{2}-b_{1}^{\dagger} b_{1} .
\end{gathered}
$$

Obtemos o hamiltoniano de Jaynes-Cummings na representação de Schrödinger :

$$
H_{J C}=H_{A}+H_{C}+H_{I N T},
$$

com:

$$
\begin{gathered}
H_{I N T}=e^{-i H_{0} t / \hbar} H_{I} e^{i H_{0} t / \hbar}=\hbar g\left[\sigma_{+} a+\sigma_{-} a^{\dagger}\right], \\
H_{A}=\hbar \omega_{1} b_{1}^{\dagger} b_{1}+\hbar \omega_{2} b_{2}^{\dagger} b_{2}=\frac{\hbar \omega_{0} \sigma_{z}}{2} \\
H_{C}=\sum_{\vec{k}, \beta} \hbar \omega_{\vec{k}, \beta} a_{\vec{k}, \beta}^{\dagger} a_{\vec{k}, \beta}=\hbar \omega a^{\dagger} a .
\end{gathered}
$$

Em que, na Eq.(3.21) definimos o ponto de energia zero em $\hbar\left(\omega_{1}+\omega_{2}\right) / 2$, ou seja, $E_{2}=$ $-E_{1}=1 / 2 \hbar \omega_{0}$, e na Eq.(3.22) omitimos o termo $\hbar \omega / 2$ por simplicidade. O hamiltoniano de Jaynes-Cummings obtido através da aproximação de dipolo elétrico e da RWA, descreve a troca de um quantum de energia entre o átomo de dois níveis e o modo da cavidade. A dinâmica do modelo de Jaynes-Cummings, pode ser estudada com a determinação dos estados estacionarios do hamiltoniano de Jaynes-Cummings. Esses autoestados são chamados os estados "vestidos". (56) Em termos dos estados número do campo, o termo de interação $\sigma_{+} a+\sigma_{-} a^{\dagger}$ do $H_{J C}$ gera somente transições do tipo $|e\rangle|n\rangle \leftrightarrow|g\rangle|n+1\rangle$ ou $|e\rangle|n-1\rangle \leftrightarrow|g\rangle|n\rangle$, sendo $|e\rangle$ o estado excitado e $|g\rangle$ o estado fundamental do átomo de dois níveis. Portanto, para um $n$ dado, definimos:

$$
\begin{gathered}
\left|\psi_{1 n}\right\rangle=|e\rangle|n\rangle, \\
\left|\psi_{2 n}\right\rangle=|g\rangle|n+1\rangle
\end{gathered}
$$

usando esta base obtemos a representação matricial do $H_{J C}$ da seguinte forma:

$$
H^{(n)}=\hbar\left(\begin{array}{cc}
\omega n+\omega_{0} / 2 & g \sqrt{n+1} \\
g \sqrt{n+1} & \omega(n+1)-\omega_{0} / 2
\end{array}\right) .
$$

Logo, diagonalizando a matriz da Eq.(3.25) obtemos como resultado as autoenergias:

$$
E_{ \pm}(n)=\left(n+\frac{1}{2}\right) \hbar \omega \pm \hbar \Omega_{n}(n)
$$


onde:

$$
\Omega_{n}(\Delta)=\sqrt{\Delta^{2}+4 g^{2}(n+1)}
$$

é a frequência de Rabi, a qual inclui agora os efetos da dessintonia, $\Delta=\omega_{0}-\omega$, entre a transição atômica e o modo da cavidade. Os autoestados associados com $E_{ \pm}(n)$ são:

$$
\begin{gathered}
|n,+\rangle=\cos \left(\Phi_{n} / 2\right)\left|\psi_{1 n}\right\rangle+\sin \left(\Phi_{n} / 2\right)\left|\psi_{2 n}\right\rangle, \\
|n,-\rangle=-\sin \left(\Phi_{n} / 2\right)\left|\psi_{1 n}\right\rangle+\cos \left(\Phi_{n} / 2\right)\left|\psi_{2 n}\right\rangle .
\end{gathered}
$$

Com:

$$
\begin{aligned}
& \sin \left(\Phi_{n} / 2\right)=\frac{1}{\sqrt{2}}\left[\frac{\Omega_{n}(\Delta)-\Delta}{\Omega_{n}(\Delta)}\right]^{1 / 2}, \\
& \cos \left(\Phi_{n} / 2\right)=\frac{1}{\sqrt{2}}\left[\frac{\Omega_{n}(\Delta)+\Delta}{\Omega_{n}(\Delta)}\right]^{1 / 2} .
\end{aligned}
$$

Os autoestados $|n, \pm\rangle$ são chamdos de estados vestidos, estos estados têm a peculiaridade de ser emaranhados, devido a que no processo de interação átomo-campo, não existe um estado do átomo ou do fóton bem definido. Além do mais, essa abordagem nos permite deduzir dois regimes importantes a saber: o regime ressonante, o qual caracteriza-se quando a dessintonia $\Delta=0$, ou seja, a frequência atômica é igual à frequência do modo da cavidade. Portanto da Eq.(3.27) temos que a frequência de Rabi nesse regime fica $\Omega_{n}(0)=2 g \sqrt{n+1}$. O outro regime é o dispersivo caracterizado por $\Omega_{n}(0) \ll \Delta$, assim que, como a dessintonia é suficientemente grande para que a transição do átomo esteja fora da ressonância com a frequência do modo da cavidade, espera-se que os efeitos da interação átomo-campo sejam enfraquecidos. O hamiltoniano efetivo dessa interação será derivado na próxima seção.

\subsection{Hamiltonianos Efetivos para interação dispersiva}

Uma variação importante do modelo de Jaynes-Cummings original, apresenta-se quando existe uma dessintonia suficientemente grande entre a transições atômicas e a frequência do campo da cavidade, de tal modo que as transições diretas não acontecem, entretanto, surgem interações dispersivas do campo da cavidade com a transições atômicas. Portanto, nessa versão do modelo de Jaynes-Cummings, precisamos obter um hamiltoniano efetivo $\left(H_{E F}\right)$ para sistemas que interagem dispersivamente, ou seja, um hamiltoniano que proporciona um melhor entendimento dos processos físicos significativos para a dinâmica do sistema. 
Para a derivação do $H_{E F}$ utilizaremos o método proposto por James e Jerke (57), o qual combina as aproximações de Markov e de ondas girantes com o processo de iteração. O método consiste em fazer uma expansão perturbativa na equação de Schrödinger, que na representação de interação escreve-se como:

$$
i \hbar \frac{\partial|\psi(t)\rangle}{\partial t}=H_{I}(t)|\psi(t)\rangle
$$

Essa equação tem a seguinte solução:

$$
|\psi(t)\rangle=|\psi(0)\rangle+\frac{1}{i \hbar} \int_{0}^{t} H_{I}\left(t^{\prime}\right)\left|\psi\left(t^{\prime}\right)\right\rangle d t^{\prime},
$$

logo, substituindo a Eq.(3.33) na Eq.(3.32), temos:

$$
|\psi(t)\rangle=H_{I}(t)|\psi(0)\rangle+\frac{1}{i \hbar} \int_{0}^{t} H_{I}(t) H_{I}\left(t^{\prime}\right)\left|\psi\left(t^{\prime}\right)\right\rangle d t^{\prime} .
$$

Considerando que os termos que compõem o hamiltoniano $H_{I}(t)$ são altamente oscilantes, podemos via RWA desprezar o primeiro termo do lado direito da Eq.(3.34) e usando a aproximação markoviana para o segundo termo, obtemos:

$$
i \hbar \frac{\partial|\psi(t)\rangle}{\partial t} \approx H_{E F}(t)|\psi(t)\rangle
$$

Onde:

$$
H_{E F}(t)=\frac{1}{i \hbar} H_{I}(t) \int_{0}^{t} H_{I}\left(t^{\prime}\right) d t^{\prime} .
$$

Frequentemente trata-se o caso de sistemas submetidos a uma perturbação com dependência temporal harmônica. Exemplo disso incluem um sistema atômico interagindo com um ou mais feixes de laser. Portanto, para obter o $H_{E F}(t)$ neste caso, consideraremos que o $H_{I}(t)$ é da forma:

$$
H_{I}(t)=\sum_{n=1}^{N}\left(h_{n} e^{-i \nu_{n} t}+h_{n}^{\dagger} e^{i \nu_{n} t}\right)
$$

obtendo:

$$
H_{E F}(t)=\frac{1}{2 \hbar} \sum_{m, n=1}^{N}\left(\frac{1}{\nu_{m}}+\frac{1}{\nu_{n}}\right)\left[h_{m}^{\dagger}, h_{n}\right] e^{i\left(\nu_{m}-\nu_{n}\right) t}
$$

Agora usaremos a técnica acima apresentada, para obter o hamiltoniano de JaynesCummings no contexto de interação dispersiva e no regime de acoplamento fraco, ou seja, $g \ll \Delta=\omega_{0}-\omega$. Então, o hamiltoniano da Eq.(3.15), na representação de interação escreve-se como:

$$
H_{I}(t)=\hbar g\left[\sigma_{+} a e^{i \Delta t}+\sigma_{-} a^{\dagger} e^{-i \Delta t}\right]
$$


se comparamos as equações (3.37) e (3.39), temos $h_{1}=\hbar g \sigma_{-} a^{\dagger}, h_{1}^{\dagger}=\hbar g \sigma_{+} a$ e $\nu_{1}=\Delta$. Portanto, da Eq.(3.38) obtemos o hamiltoniano efetivo desejado:

$$
\begin{aligned}
H_{E F}(t) & =\frac{1}{\hbar \Delta}\left[h_{1}^{\dagger}, h_{1}\right] \\
& =\frac{\hbar g^{2}}{\Delta}\left\{\sigma_{+} \sigma_{-}+a^{\dagger} a\left(\sigma_{+} \sigma_{-}-\sigma_{-} \sigma_{+}\right)\right\} \\
& =\hbar \chi a^{\dagger} a \sigma_{z}+\hbar \chi \sigma_{+} \sigma_{-},
\end{aligned}
$$

onde $\chi=g^{2} / \Delta$. O último termo da Eq.(3.40) pode ser eliminado por meio de uma transformação unitaria, $U=\exp \left(i \chi t \sigma_{+} \sigma_{-}\right)$, já que, este termo carreia somente uma fase adicional à dinâmica do sistema.

Os hamiltonianos efetivos têm grande utilidade na engenharia, de estados tipo Einstein-Poldolsky-Rosen (EPR) (14), de estados de dois átomos maximamente emaranhados. (58) Além do mais, esse protocolo foi usado também para engenhar um modo não estacionario de uma cavidade não ideal, onde mostrou-se que a engenharia deste modo, pode proteger o estado do sistema da decoerência (59), dentre inúmeras outras aplicações. 
3.3. Hamiltonianos Efetivos para interação dispersiva 


\section{Capítulo 4}

\section{Simulação da Equação de Dirac em EQC}

\subsection{Introdução}

Os efeitos quânticos relativísticos são fundamentais para a compreensão da teoria quântica relativística, mas são difíceis de observar experimentalmente. Por conseguinte, as simulações dos efeitos quânticos relativísticos têm recebido apreciável atenção nos últimos anos. Recentemente (34), simulou-se a equação de Dirac $1+1$ D no contexto de armadilha iônica, usando um sistema constituído de um único íon de massa $M$ dentro de uma armadilha do tipo Paul, onde o íon na armadilha simula matematicamente uma partícula relativística livre (33). Neste modelo, o momento e a posição da partícula relativística correspondem às quadraturas do estado vibracional da armadilha o que foi usado para demonstrar o Zitterbewegung associado à equação de Dirac.

Motivado pelos trabalhos mencionados acima, neste capítulo demostraremos como simular o hamiltoniano de Dirac $2+1$ D e $1+1$ D para uma partícula relativística livre via EQC. Inicialmente, vamos obter o hamiltoniano associado a Dirac $2+1 \mathrm{D}$, onde utilizaremos um sistema atômico interagindo fora de ressonância com dois modos da cavidade e quatro campos clássicos. Posteriormente, mostramos como é possível obter o hamiltoniano de Dirac $1+1 \mathrm{D}$ a partir do hamiltoniano $2+1 \mathrm{D}$, e em seguida, analisarmos a validade das aproximações feitas via cálculo numérico. Finalmente estudamos o efeito relativístico Zitterbewegung no contexto da EQC e discutimos uma forma de simulá-lo.

\subsection{Hamiltoniano de Dirac via EQC}

O hamiltoniano de Dirac $2+1$ D é obtido eliminando-se uma componente espacial da equação de Dirac $3+1$ D. Neste caso, as condições entre as matrizes $\beta$ e $\alpha_{k}$ explicadas no Capitulo. 2, são satisfeitas somente com matrizes $2 \times 2$. 
Portanto, podemos construir o hamiltoniano de Dirac $2+1 \mathrm{D}\left(H_{D}^{2+1}\right)$ usando a correspondência que existe entre as matrizes $\alpha_{1}, \alpha_{2}$ e $\beta$ e as matrizes de Pauli $\sigma_{1}, \sigma_{2}$ e $\sigma_{3}$, respectivamente, obtendo:

$$
H_{D}^{2+1}=m c^{2} \sigma_{3}+c p_{1} \sigma_{1}+c p_{2} \sigma_{2}
$$

onde 1, 2 e 3 correspondem a $x, y$, e $z$, respectivamente. A expressão (4.1) descreve a dinâmica de uma partícula relativística livre em duas dimensões. Agora, o próximo passo para seguir a nossa discussão é considerar um átomo de quatro níveis no interior de uma cavidade de alto fator de qualidade, onde o átomo é submetido a interações fora de ressonância com dois modos da cavidade, de frequências $\omega_{a}$ e $\omega_{b}$, e com quatro campos clássicos de frequências $\omega_{1}, \omega_{2}, \omega_{3}$ e $\omega_{4}$. Com a finalidade de descrever este sistema, usamos a configuração ilustrada na Figura 4.1, onde apresenta-se esquematicamente a interação átomo-campo. Quando o átomo de quatro níveis interage com os modos da cavidade induzem-se transições de dipolo entre os níveis, $|g\rangle \leftrightarrow|i\rangle$ com constantes de acoplamentos $\lambda_{a}$ e $\lambda_{b}$ (escrevemos em todo esse capítulo os parâmetros que dependem do modo $\omega_{a}$ ou do modo $\omega_{b}$, com o subíndice $a$ ou $b$ ) e entre os níveis $|e\rangle \leftrightarrow|f\rangle$, com constantes de acoplamentos $\tilde{\lambda}_{a}$ e $\tilde{\lambda}_{b}$. Sendo, $|g\rangle$ o estados fundamental, $|e\rangle$ o estado excitado, $|i\rangle$ e $|f\rangle$ estados intermediários. Aqui consideramos o regime em que os modos quânticos não são ressonantes com essas transições de dipolo, consequentemente, definimos as dessintonias entre as frequências dos modos e das transições atômicas como: $\Delta_{a}=$ $\omega_{a}-\omega_{i}, \tilde{\Delta}_{a}=\left(\omega_{a}+\omega_{e}\right)-\omega_{f}, \Delta_{b}=\omega_{i}-\omega_{b}$ e $\tilde{\Delta}_{b}=\omega_{f}-\left(\omega_{b}+\omega_{e}\right)$, onde, $\omega_{i}, \omega_{e}$ e $\omega_{f}$ (com $\left.\omega_{g}=0\right)$ são as frequências de transição atômica dos níveis $|i\rangle,|e\rangle$ e $|f\rangle$, respectivamente. Conjuntamente com os campos quânticos, os campos clássicos induzem transições de dipolo (não ressonantes) entre os níveis $|e\rangle \leftrightarrow|i\rangle$ e $|g\rangle \leftrightarrow|f\rangle$, com constantes de acoplamentos $\Omega_{1}, \Omega_{3}, \Omega_{2}$ e $\Omega_{4}$ e dessintonias $\Delta_{1}=\left(\omega_{1}+\omega_{e}\right)-\omega_{i}, \Delta_{3}=\omega_{i}-\left(\omega_{3}+\omega_{e}\right), \Delta_{2}=\omega_{2}-\omega_{f}$ e $\Delta_{4}=\omega_{f}-\omega_{4}$, respectivamente. Por conseguinte, partindo do hamiltoniano total desse sistema, o qual pode ser escrito na aproximação de onda girante, como $H_{T}^{2 D}=H_{0}+V$, onde:

$$
\begin{gathered}
H_{0}=\hbar \omega_{a} a^{\dagger} a+\hbar \omega_{b} b^{\dagger} b+\hbar \omega_{e} \sigma_{e e}+\hbar \omega_{i} \sigma_{i i}+\hbar \omega_{f} \sigma_{f f}, \\
V=\hbar \lambda_{a} a \sigma_{i g}+\hbar \Omega_{1} \sigma_{i e} e^{-i \omega_{1} t}+\hbar \tilde{\lambda}_{a} a \sigma_{f e}+\hbar \Omega_{2} \sigma_{f g} e^{-i \omega_{2} t}+\hbar \lambda_{b} b^{\dagger} \sigma_{g i} \\
+\hbar \Omega_{3} \sigma_{e i} e^{i \omega_{3} t}+\hbar \tilde{\lambda}_{b} b^{\dagger} \sigma_{e f}+\hbar \Omega_{4} \sigma_{g f} e^{i \omega_{4} t}+h . c .
\end{gathered}
$$

Sendo, $H_{0}$ o hamiltoniano do campo e do átomo, $V$ o hamiltoniano de interação átomocampo, $\sigma_{k j} \equiv|k\rangle\langle j|$ (com $\left.k, j=g, e, i, f\right)$ são os operadores pseudo-spin os quais 
Figura 4.1 - Configuração de níveis atômicos necessária para gerar o Hamiltoniano de Dirac $2+1$ D.

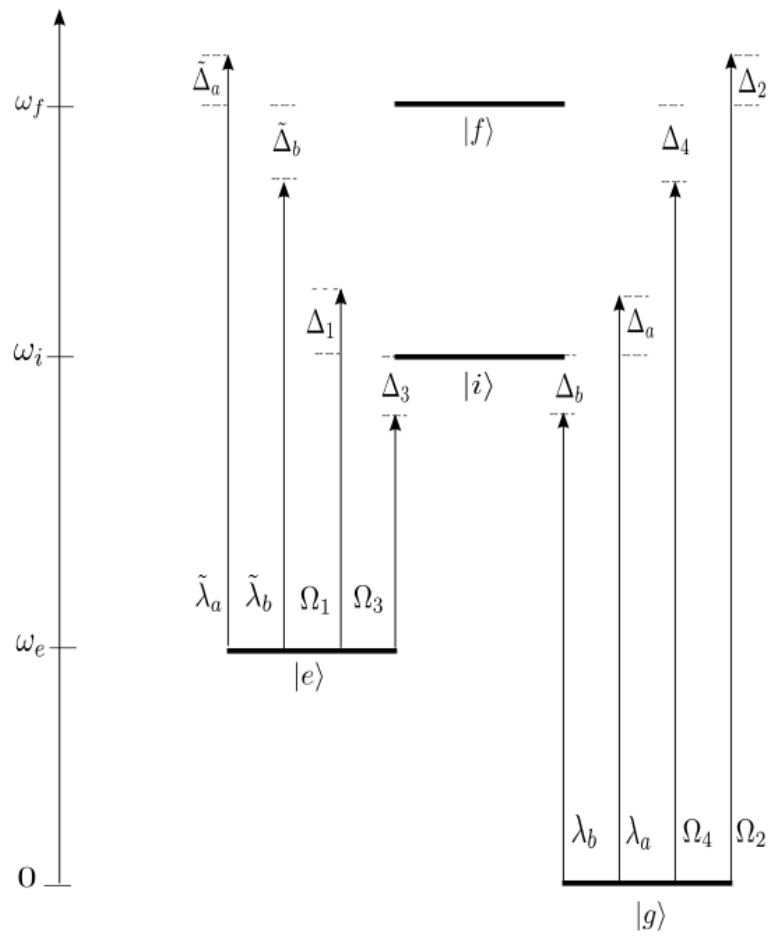

Fonte: Elaborada pelo autor.

descrevem as transições atômicas, $a^{\dagger}(a)$ e $b^{\dagger}(b)$ são os operadores de criação (aniquilação) de fótons dos modos da cavidade. Consequentemente, a partir da obtenção do $H_{T}^{2 D}$ podemos estudar a dinâmica da interação átomo-campo na representação de interação. Assim que, para obter $H_{T}^{2 D}$ na representação de interação usamos a transformação unitária $U_{0}=\exp \left(-i H_{0} t / \hbar\right)$, chegando à seguinte expressão:

$$
\begin{aligned}
V(t)= & \hbar \lambda_{a} a \sigma_{i g} e^{-i \Delta_{a} t}+\hbar \Omega_{1} \sigma_{i e} e^{-i \Delta_{1} t}+\hbar \tilde{\lambda}_{a} a \sigma_{f e} e^{-i \tilde{\Delta}_{a} t}+\hbar \Omega_{2} \sigma_{f g} e^{-i \Delta_{2} t}+\hbar \lambda_{b} b^{\dagger} \sigma_{g i} e^{-i \Delta_{b} t} \\
& +\hbar \Omega_{3} \sigma_{e i} e^{-i \Delta_{3} t}+\hbar \tilde{\lambda}_{b} b^{\dagger} \sigma_{e f} e^{-i \tilde{\Delta}_{b} t}+\hbar \Omega_{4} \sigma_{g f} e^{-i \Delta_{4} t}+h . c .
\end{aligned}
$$

Por outro lado, como estamos interessados em estudar o regime dispersivo, é necessário assumir que as condições $\sqrt{\bar{n}_{\alpha}+1} \lambda_{\alpha} \ll \Delta_{\alpha}, \sqrt{\bar{n}_{\alpha}+1} \tilde{\lambda}_{\alpha} \ll \tilde{\Delta}_{\alpha}$ e $\Omega_{s} \ll \Delta_{s}$ (com $s=1,2,3,4$ e $\alpha=a, b)$, sejam válidas, sendo $\bar{n}_{\alpha}$ o número médio de fótons. Neste contexto como o hamiltoniano $V(t)$ tem termos altamente oscilantes, utilizamos um hamiltoniano efetivo $H_{E F}^{2 D}$ de segunda ordem, seguindo o método descrito no Capítulo 3. Em vista disso, para determinar o hamiltoniano efetivo da Eq.(4.4) utilizamos um hamiltoniano com dependência temporal harmônica e frequências, $\nu_{n}$, da seguinte forma: 


$$
\tilde{V}(t)=\sum_{n=1}^{N}\left(h_{n} e^{-i \nu_{n} t}+h_{n}^{\dagger} e^{i \nu_{n} t}\right)
$$

onde $h_{n}$ e $h_{n}^{\dagger}$ são operadores independentes do tempo. Agora, comparando os hamiltonianos $V(t)$ e $\tilde{V}(t)$, e usando a relação:

$$
H_{E F}=\sum_{m, n=1}^{N} \frac{1}{2 \hbar}\left(\frac{1}{\nu_{m}}+\frac{1}{\nu_{n}}\right)\left[h_{m}^{\dagger}, h_{n}\right] e^{i\left(\nu_{m}-\nu_{n}\right) t}
$$

obtemos:

$$
\begin{aligned}
H_{E F}^{2 D}= & \hbar \delta_{a} a^{\dagger} a\left(\sigma_{e e}+\sigma_{g g}\right)-\hbar \delta_{b} b^{\dagger} b\left(\sigma_{e e}+\sigma_{g g}\right)+\hbar \frac{\Omega_{1}^{2}}{\Delta_{1}} \sigma_{e e}+\hbar \frac{\Omega_{2}^{2}}{\Delta_{2}} \sigma_{g g}-\hbar \frac{\Omega_{3}^{2}}{\Delta_{3}} \sigma_{e e}-\hbar \frac{\Omega_{4}^{2}}{\Delta_{4}} \sigma_{g g} \\
& +\frac{\hbar}{2} \delta_{a}\left(a^{\dagger} \sigma_{g e} e^{-i \delta_{a} t}+a^{\dagger} \sigma_{e g} e^{-i \delta_{a} t}+\text { h.c. }\right) \\
& +\frac{\hbar}{2} \delta_{b}\left(b \sigma_{g e} e^{-i \delta_{b} t}-b \sigma_{e g} e^{-i \delta_{b} t}+\text { h.c. }\right)
\end{aligned}
$$

onde, ficamos com os termos que cumprem as seguintes condições:

$$
\begin{aligned}
& \left(\frac{1}{\Delta_{a}}+\frac{1}{\Delta_{1}}\right) \lambda_{a} \Omega_{1}=\delta_{a} \\
& \left(\frac{1}{\tilde{\Delta}_{a}}+\frac{1}{\Delta_{2}}\right) \tilde{\lambda}_{a} \Omega_{2}=\delta_{a} \\
& \left(\frac{1}{\Delta_{b}}+\frac{1}{\Delta_{3}}\right) \lambda_{b} \Omega_{3}=\delta_{b} \\
& \left(\frac{1}{\tilde{\Delta}_{b}}+\frac{1}{\Delta_{4}}\right) \tilde{\lambda}_{b} \Omega_{4}=\delta_{b}
\end{aligned}
$$

com, $\Delta_{1}-\Delta_{a}=\Delta_{2}-\tilde{\Delta}_{a}=\delta_{a}, \Delta_{3}-\Delta_{b}=\Delta_{4}-\tilde{\Delta}_{b}=\delta_{a}, \delta_{\alpha}=\lambda_{\alpha}^{2} / \Delta_{\alpha}=\tilde{\lambda}_{\alpha}^{2} / \tilde{\Delta}_{\alpha}$, (sendo $\delta_{\alpha}$ sempre positivos). As condições anteriores nos permite desprezar os efeitos dos termos altamente oscilantes, que em media temporal não contribuem efetivamente para a dinâmica do sistema (aproximação de onda girante). Portanto na Eq.(4.7), utilizamos aproximação adiabática nos estados intermediários $|i\rangle$ e $|f\rangle$, ou seja, impondo-se que estes estados não são populados.

Através do hamiltoniano da Eq.(4.7), podemos obter o hamiltoniano de Dirac $2+1$ D. Para visualizarmos e compreendermos melhor tal analogia, usamos as seguintes relações:

$$
\begin{gathered}
\sigma_{g g}=\frac{I-\sigma_{3}}{2}, \\
\sigma_{e e}=\frac{I+\sigma_{3}}{2},
\end{gathered}
$$


sendo:

$$
\begin{gathered}
\sigma_{3}=\sigma_{e e}-\sigma_{g g}=\left(\begin{array}{cc}
1 & 0 \\
0 & -1
\end{array}\right), \\
I=\sigma_{e e}+\sigma_{g g}=\left(\begin{array}{ll}
1 & 0 \\
0 & 1
\end{array}\right) .
\end{gathered}
$$

Substituindo as relações (4.12) e (4.13) na Eq.(4.7), temos:

$$
\begin{aligned}
H_{E F}^{2 D} & =\hbar \delta_{a} a^{\dagger} a-\hbar \delta_{b} b^{\dagger} b+\zeta_{-} I+\zeta_{+} \sigma_{3}+\frac{\hbar}{2} \delta_{a}\left(a^{\dagger} \sigma_{g e} e^{-i \delta_{a} t}+a^{\dagger} \sigma_{e g} e^{-i \delta_{a} t}+h . c .\right) \\
& +\frac{\hbar}{2} \delta_{b}\left(b^{\dagger} \sigma_{e g} e^{i \delta_{b} t}-b^{\dagger} \sigma_{g e} e^{i \delta_{b} t}+\text { h.c. }\right)
\end{aligned}
$$

onde:

$$
\left.\zeta_{ \pm}=\frac{\hbar}{2}\left\{\frac{\Omega_{1}^{2}}{\Delta_{1}} \mp \frac{\Omega_{2}^{2}}{\Delta_{2}} \pm \frac{\Omega_{4}^{2}}{\Delta_{4}}-\frac{\Omega_{3}^{2}}{\Delta_{3}}\right)\right\}
$$

Por conseguinte, eliminando os termos diagonais:

$$
H=\hbar \delta_{a} a^{\dagger} a+\hbar \delta_{b} b^{\dagger} b+\zeta_{-} I
$$

contemplados no hamiltoniano efetivo da Eq.(4.16), por meio da transformação unitária:

$$
H_{E F}^{\prime 2 D}=e^{i H t / \hbar} H_{E F}^{2 D} e^{-i H t / \hbar}-H
$$

obtemos o resultado a seguir:

$$
H_{E F}^{\prime 2 D}=\zeta_{+} \sigma_{3}+\frac{\hbar}{2} \delta_{a}\left(a^{\dagger}+a\right)\left(\sigma_{g e}+\sigma_{e g}\right)+\frac{\hbar}{2} \delta_{b}\left(b^{\dagger}-b\right)\left(\sigma_{e g}-\sigma_{g e}\right),
$$

logo, usando a transformação unitária $U=\exp \left\{-i \frac{\pi}{2} a^{\dagger} a\right\}$ temos:

$$
H_{E F}^{\prime^{2 D}}=\zeta_{+} \sigma_{3}+i \frac{\hbar}{2} \delta_{a}\left(a^{\dagger}-a\right)\left(\sigma_{g e}+\sigma_{e g}\right)+i^{2} \frac{\hbar}{2} \delta_{b}\left(b^{\dagger}-b\right)\left(\sigma_{g e}-\sigma_{e g}\right)
$$

Comparando a Eq.(4.21) com a Eq.(4.1), podemos notar a correspondência entre os seguintes parâmetros:

$$
\begin{gathered}
m c^{2}:=\zeta_{+}, \\
c:=R_{a} \delta_{a}=R_{b} \delta_{b}, \\
p_{1}=\frac{i \hbar}{2 R_{a}}\left(a^{\dagger}-a\right),
\end{gathered}
$$




$$
\begin{gathered}
p_{2}=\frac{i \hbar}{2 R_{b}}\left(b^{\dagger}-b\right), \\
\sigma_{1}=\sigma_{g e}+\sigma_{e g}, \\
\sigma_{2}=i\left(\sigma_{g e}-\sigma_{e g}\right),
\end{gathered}
$$

onde incorporamos as constantes $R_{\alpha}=\sqrt{\hbar / 2 m_{e} \omega_{\alpha}}$, para ajustar as unidades dos operadores $p_{1}$ e $p_{2}$. O hamiltoniano de Dirac de mais baixa dimensão, é obtido eliminando-se um modo da cavidade e dois campos clássicos da Eq.(4.21). Por conseguinte, temos dois métodos para obter hamiltoniano de Dirac $1+1 \mathrm{D}$ a partir do sistema anterior. O primeiro método consiste em desligar ( ver Figura 4.1 ) o modo de frequência $\omega_{b}$ e os campos clássicos de frequências $\omega_{3}$ e $\omega_{4}$, desta maneira, o novo sistema preparado para a simulação estará conformado por dois campos clássicos $\left(\omega_{1}\right.$ e $\left.\omega_{2}\right)$, um modo da cavidade $\left(\omega_{a}\right)$ e quatro níveis $(|g\rangle,|e\rangle,|i\rangle,|f\rangle)$. O segundo método baseia-se em assumir no primeiro método que os níveis $|g\rangle$ e $|e\rangle$ são quase degenerados o que nos permite omitir o nível $|f\rangle$.

Nesta seção vamos discutir o segundo método, já que um sistema de três níveis reduz um pouco mais as exigências experimentais. A Figura 4.2 ilustra nosso novo sistema de três níveis, onde o estado fundamental $|g\rangle$ e o excitado $|e\rangle$ são acoplados ao estados intermediário $|i\rangle$ por meio do modo da cavidade, de frequência $\omega_{a}$ que possibilita as transições de dipolo $|g\rangle \leftrightarrow|i\rangle$ e $|e\rangle \leftrightarrow|i\rangle$, com constantes de acoplamentos $\lambda_{a}$ e $\tilde{\lambda}_{a}$, respectivamente. Aqui consideramos novamente o regime em que a frequência do modo da cavidade não é ressonante com as frequências de transições atômicas. Portanto, definimos as dessintonias $\Delta_{a}=\omega_{a}-\omega_{i}$ e $\tilde{\Delta}_{a}=\left(\omega_{a}+\omega_{e}\right)-\omega_{i}$, onde $\omega_{i}, \omega_{e}$ são as frequências de transição atômica dos níveis $|i\rangle,|e\rangle$. Simultaneamente, os dois campos clássicos das frequências, $\omega_{1}$ e $\omega_{2}$, induzem transições não ressonantes entre os níveis $|e\rangle \leftrightarrow|i\rangle$ e $|g\rangle \leftrightarrow|i\rangle$ com constantes de acoplamentos $\Omega_{1}, \tilde{\Omega}_{2}, \Omega_{2}, \tilde{\Omega}_{1}$ e dessintonias $\Delta_{1}=\left(\omega_{1}+\omega_{e}\right)-\omega_{i}, \tilde{\Delta}_{2}=\left(\omega_{2}+\omega_{e}\right)-\omega_{i}$, $\Delta_{2}=\omega_{2}-\omega_{i}, \tilde{\Delta}_{1}=\omega_{i}-\omega_{1}$, respectivamente. Novamente, o primeiro passo para iniciar nosso tratamento consiste em escrever o hamiltoniano total deste sistema, o qual é dado por $H_{T}^{1 D}=H_{0}^{\prime}+V^{\prime}$, onde:

$$
\begin{gathered}
H_{0}^{\prime}=\hbar \omega_{a} a^{\dagger} a+\hbar \omega_{e} \sigma_{e e}+\hbar \omega_{i} \sigma_{i i} \\
V^{\prime}=\hbar \lambda_{a} a \sigma_{i g}+\hbar \Omega_{1} \sigma_{i e} e^{-i \omega_{1} t}+\hbar \tilde{\lambda}_{a} a \sigma_{i e}+\hbar \Omega_{2} \sigma_{i g} e^{-i \omega_{2} t} \\
+\hbar \tilde{\Omega}_{1} \sigma_{g i} e^{i \omega_{1} t}+\hbar \tilde{\Omega}_{2} \sigma_{i e} e^{-i \omega_{2} t}+\text { h.c. }
\end{gathered}
$$

Os operadores que se encontram nessas expressões estão definidos na seção anterior. Agora 
Figura 4.2 - Configuração de níveis atômicos, usada para gerar o Hamiltoniano de Dirac $1+1$ D.

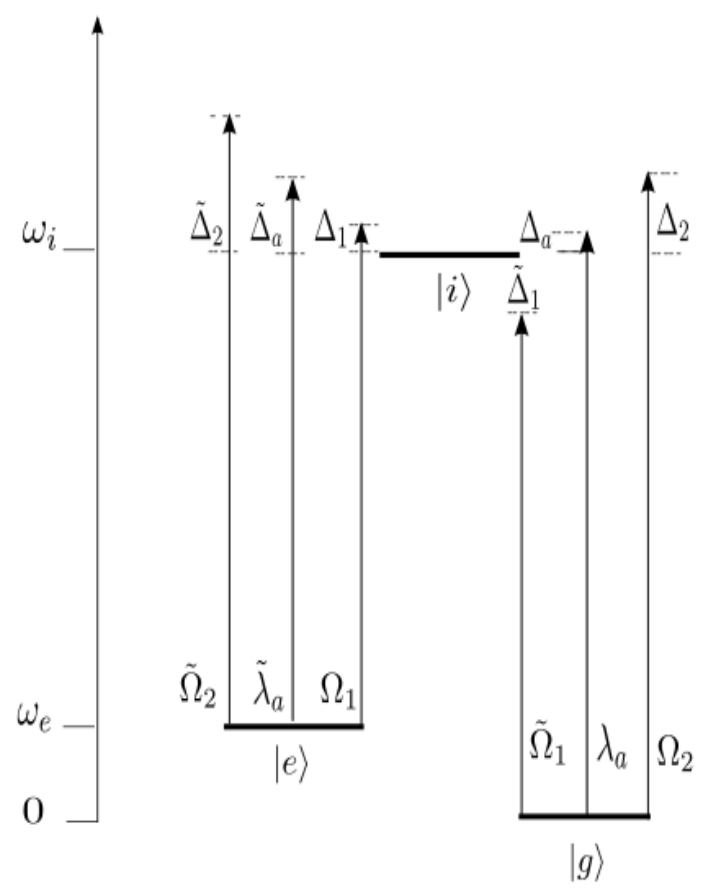

Fonte: Elaborada pelo autor.

$H_{T}^{1 D}$ na representação de interação torna-se:

$$
\begin{aligned}
V^{\prime}(t)= & \hbar \lambda_{a} a \sigma_{i g} e^{-i \Delta_{a} t}+\hbar \Omega_{1} \sigma_{i e} e^{-i \Delta_{1} t}+\hbar \tilde{\lambda}_{a} a \sigma_{i e} e^{-i \tilde{\Delta}_{a} t}+\hbar \Omega_{2} \sigma_{i g} e^{-i \Delta_{2} t} \\
& +\hbar \tilde{\Omega}_{1} \sigma_{g i} e^{-i \tilde{\Delta}_{1} t}+\hbar \tilde{\Omega}_{2} \sigma_{i e} e^{-i \tilde{\Delta}_{2} t}+h . c . .
\end{aligned}
$$

Como a Eq.(4.30) tem termos altamente oscilantes, podemos obter o hamiltoniano efetivo para $V^{\prime}(t)$ no regime dispersivo, no qual consideramos $\sqrt{\bar{n}_{a}+1} \lambda_{a} \ll \Delta_{a}, \sqrt{\bar{n}_{a}+1} \tilde{\lambda}_{a} \ll$ $\tilde{\Delta}_{a}, \Omega_{m} \ll \Delta_{m}$ e $\tilde{\Omega}_{m} \ll \tilde{\Delta}_{m}$ (com $\left.m=1,2\right)$. Portanto, comparando a Eq.(4.30) com a Eq.(4.5) e usando a Eq.(4.6) obtemos:

$$
\begin{aligned}
H_{E F}^{1 D}= & \hbar \frac{\lambda_{a}^{2}}{\Delta_{a}}\left(a^{\dagger} a \sigma_{g g}-a a^{\dagger} \sigma_{i i}\right)+\hbar \frac{\Omega_{1}^{2}}{\Delta_{1}}\left(\sigma_{e e}-\sigma_{i i}\right)+\hbar \frac{\tilde{\lambda}_{a}^{2}}{\tilde{\Delta}_{a}}\left(a^{\dagger} a \sigma_{e e}-a a^{\dagger} \sigma_{f f}\right) \\
& +\hbar \frac{\Omega_{2}^{2}}{\Delta_{2}}\left(\sigma_{e e}-\sigma_{f f}\right)+\hbar \frac{\tilde{\Omega}_{1}^{2}}{\tilde{\Delta}_{1}}\left(\sigma_{i i}-\sigma_{g g}\right)+\hbar \frac{\tilde{\Omega}_{2}^{2}}{\tilde{\Delta}_{2}}\left(\sigma_{e e}-\sigma_{i i}\right) \\
& +\frac{\hbar}{2}\left(\frac{1}{\Delta_{a}}+\frac{1}{\Delta_{1}}\right) \lambda_{a} \Omega_{1}\left(a^{\dagger} \sigma_{g e} e^{-i \delta t}+a \sigma_{e g} e^{i \delta t}\right) \\
& +\frac{\hbar}{2}\left(\frac{1}{\tilde{\Delta}_{a}}+\frac{1}{\Delta_{1}}\right) \tilde{\lambda}_{a} \Omega_{2}\left(a^{\dagger} \sigma_{e g} e^{-i \delta t}+a \sigma_{g e} e^{i \delta t}\right)
\end{aligned}
$$


sendo $\Delta_{1}-\Delta_{a}=\delta, \Delta_{2}-\tilde{\Delta}_{a}=\delta$. O hamiltoniano efetivo da Eq.(4.31) foi obtido via aproximação de onda girante, onde impomos as seguintes condições:

$$
\begin{aligned}
& \left(\frac{1}{\Delta_{a}}+\frac{1}{\Delta_{1}}\right) \lambda_{a} \Omega_{1}=\delta, \\
& \left(\frac{1}{\tilde{\Delta}_{a}}+\frac{1}{\Delta_{2}}\right) \tilde{\lambda}_{a} \Omega_{2}=\delta .
\end{aligned}
$$

Em consequência, utilizando aproximação adiabática no estado intermediário, $|i\rangle$ e as relações entre a matriz de Pauli $\sigma_{z}$ e os operadores atômicos, definidas nas Eqs. (4.12), (4.13) e (4.14), é fácil ver que o hamiltoniano da Eq.(4.31) escreve-se como:

$$
H_{E F}^{1 D}=\hbar \delta a^{\dagger} a+\zeta_{-}^{\prime} I+\zeta_{+}^{\prime} \sigma_{z}+\frac{\hbar}{2} \delta\left(a^{\dagger} \sigma_{e g} e^{-i \delta t}+a^{\dagger} \sigma_{g e} e^{-i \delta t}+h . c .\right)
$$

onde:

$$
\left.\zeta_{ \pm}^{\prime}=\frac{\hbar}{2}\left\{\frac{\Omega_{1}^{2}}{\Delta_{1}}+\frac{\tilde{\Omega}_{2}^{2}}{\tilde{\Delta}_{2}} \mp \frac{\Omega_{2}^{2}}{\Delta_{2}} \pm \frac{\tilde{\Omega}_{1}^{2}}{\tilde{\Delta}_{1}}\right)\right\}
$$

e consideramos que:

$$
\delta=\frac{\lambda_{a}^{2}}{\Delta_{a}}=\frac{\tilde{\lambda}_{a}^{2}}{\tilde{\Delta}_{a}} .
$$

Se aplicamos na Eq.(4.34) as transformações

$$
H_{E F}^{\prime 1 D}=e^{i H^{\prime} t / \hbar} H_{E F}^{1 D} e^{-i H^{\prime} t / \hbar}-H^{\prime}
$$

com:

$$
H^{\prime}=\hbar \delta a^{\dagger} a+\zeta_{-}^{\prime} I
$$

e $\operatorname{logo} U=\exp \left\{-i \frac{\pi}{2} a^{\dagger} a\right\}$ obtemos:

$$
H_{E F}^{\prime^{1 D}}=\zeta_{+}^{\prime} \sigma_{3}+i \frac{\hbar}{2} \delta\left(a^{\dagger}-a\right)\left(\sigma_{g e}+\sigma_{e g}\right) .
$$

Comparando esta equação com a Eq.(4.1) temos:

$$
\begin{gathered}
c:=R_{a} \delta, \\
m c^{2}:=\zeta_{+}^{\prime}, \\
p_{1}=\frac{i \hbar}{2 R_{a}}\left(a^{\dagger}-a\right),
\end{gathered}
$$




$$
\begin{gathered}
\sigma_{1}=\left(\sigma_{g e}+\sigma_{e g}\right), \\
\sigma_{2}=0 .
\end{gathered}
$$

A interessante analogia das Eqs. (4.21) e (4.39) com a Eq.(4.1), mostra que nosso sistema permite manipular e controlar por meio das forças de acoplamentos dos campos, os valores da velocidade da luz e a energia de repouso da partícula relativística livre de Dirac simulada. Portanto, a dinâmica de um elétron no formalismo da mecânica quântica relativística, pode ser simulada usando experimentos em EQC. Não obstante, a simulação dos efeitos quânticos relativísticos não demostram a existência deles, mas permite com as mesmas leis quânticas explorar as correspondências e diferenças entre tais sistemas físicos.

\subsection{Validade das aproximações}

Para analisar via cálculo numérico a validade das aproximações feitas para obter o hamiltoniano de Dirac 2+1 D comparamos a evolução de um dado estado inicial do nosso sistema considerando o hamiltonianos total na representação de interação da Eq. (4.4) e o hamiltoniano efetivo da Eq. (4.21). Portanto, considerando o átomo preparado no estado inicial $|e\rangle$, os modos da cavidade no estado $|0,0\rangle$ ( $a$ e $b$ no vácuo), as forças de acoplamen-

$\operatorname{tos} \lambda_{\alpha}, \tilde{\lambda}_{\alpha}$, e $\Omega_{s}$ aproximadamente iguais a $\lambda=10^{5} \mathrm{~Hz}$ (usaremos esse parâmetro ao longo deste capítulo ) e as dessintonias $\Delta_{\alpha} \sim \Delta_{1 \text { ou } 3} \sim 38 \lambda, \tilde{\Delta}_{a} \sim \Delta_{2} \sim 50 \lambda$ e $\tilde{\Delta}_{b} \sim \Delta_{4} \sim 75 \lambda$, obtemos a Figura 4.3. A Fig.(4.3-a) ilustra a probabilidade do átomo manter-se no nível $|e\rangle\left(P_{e}\right)$, de ir para o nível $|g\rangle\left(P_{g}\right)$ e a soma das probabilidades $P=P_{e}+P_{g}$ versus o tempo de interação adimensional $g t=2 \pi$. A Fig.(4.3-b) mostra as populações dos estados $|0,0\rangle$ $\left(P_{0,0}\right)$ e $|1,1\rangle\left(P_{1,1}\right)$ versus $g t$. Através dos gráficos (4.3-a) e (4.3-b) verificamos um casamento muito bom entre as curvas obtidas a partir do Hamiltoniano efetivo (4.21) e o sem aproximação (4.4). Portanto, o erro devido à aproximações feitas torna-se praticamente desprezível confirmando que tanto a dinâmica do campo como do átomo do hamiltoniano (4.4) é análoga ao hamiltoniano efetivo (4.21). Desta mesma maneira, examinamos a validade das aproximações feitas para obter o hamiltoniano de Dirac $1+1 \mathrm{D}$, comparando a evolução de um dado estado inicial do nosso sistema considerando o hamiltonianos total da Eq. (4.29) e o hamiltoniano efetivo da Eq. (4.39), obtemos as Figuras (4.4-a) e (4.4b). Considerando o sistema átomo-campo preparado no estado inicial $|e, 0\rangle$, as forças de acoplamentos com valores aproximados a $\lambda$ e $\Delta_{a} \sim \Delta_{1} \sim 20 \lambda, \tilde{\Delta}_{a} \sim \Delta_{2} \sim 80 \lambda, \tilde{\Delta}_{1} \sim 40 \lambda$ e $\tilde{\Delta}_{2} \sim 140 \lambda$.

Por consequência, de acordo com nossas simulações numéricas a validade das aproximações feitas anteriormente são aceitáveis, já que a dinâmica do átomo e o campo dos 
hamiltonianos efetivos e os hamiltoniano totais é praticamente igual.

Figura 4.3 - Probabilidades obtidas a partir do Hamiltoniano efetivo (4.21) (curvas contínuas) e do Hamiltoniano sem aproximação (4.4) (curvas pontilhadas).

(a) $P_{e}, P_{g}$ e $P$ e (b) $P_{0,0}, P_{1,1}$.

(a)

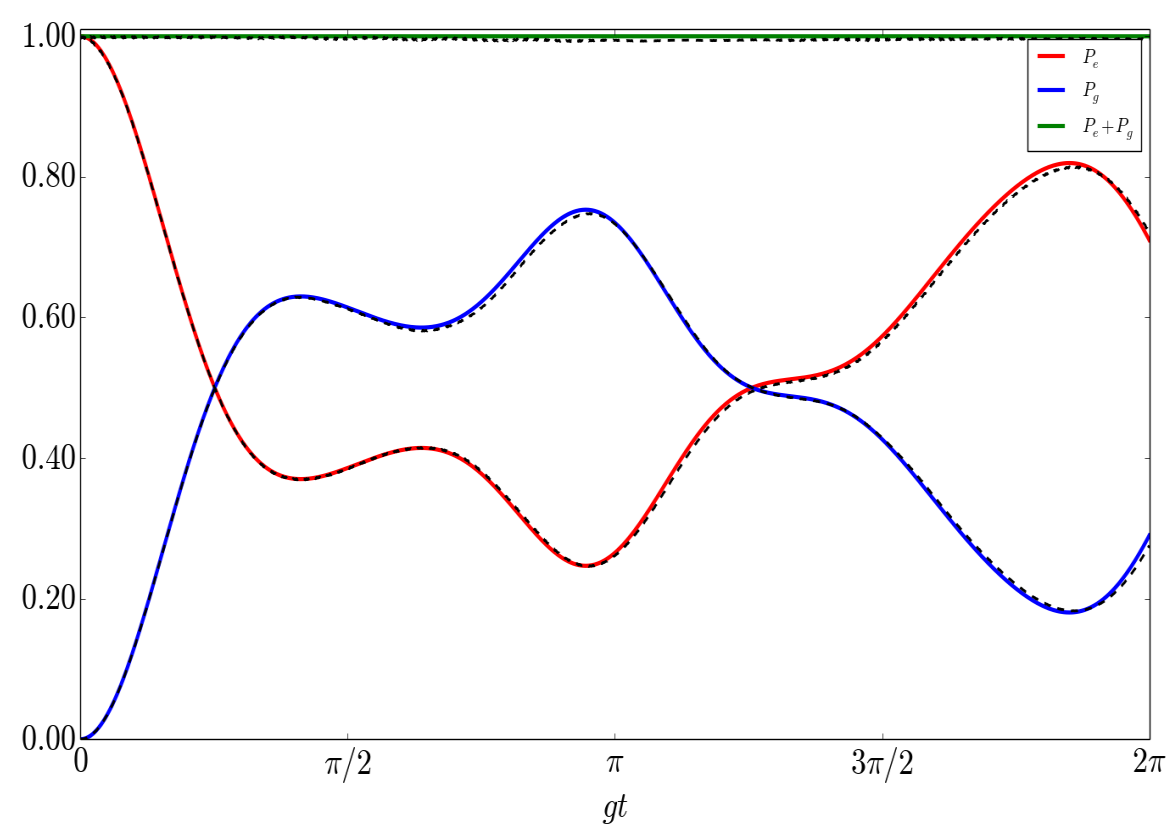

(b)

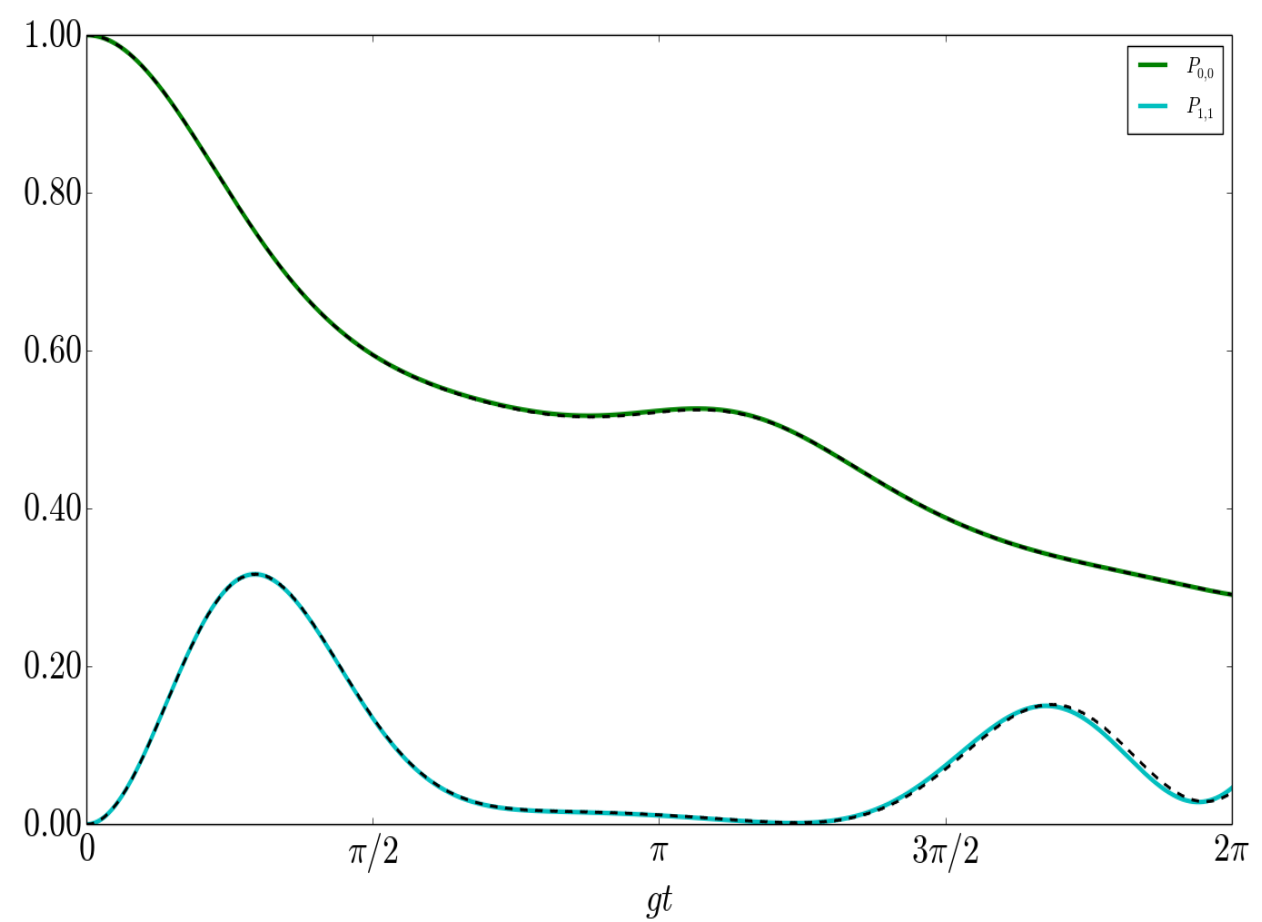

Fonte: Elaborada pelo autor. 
Figura 4.4 - Probabilidades obtidas a partir do Hamiltoniano efetivo (3.39) (curvas contínuas) e do Hamiltoniano sem aproximação (3.29) (curvas pontilhadas). (a) $P_{e}, P_{g}$ e $P$ e (b) Populações do estado do campo $|0\rangle\left(P_{0}\right)$ e $|1\rangle$ $\left(P_{1}\right)$.

(a)

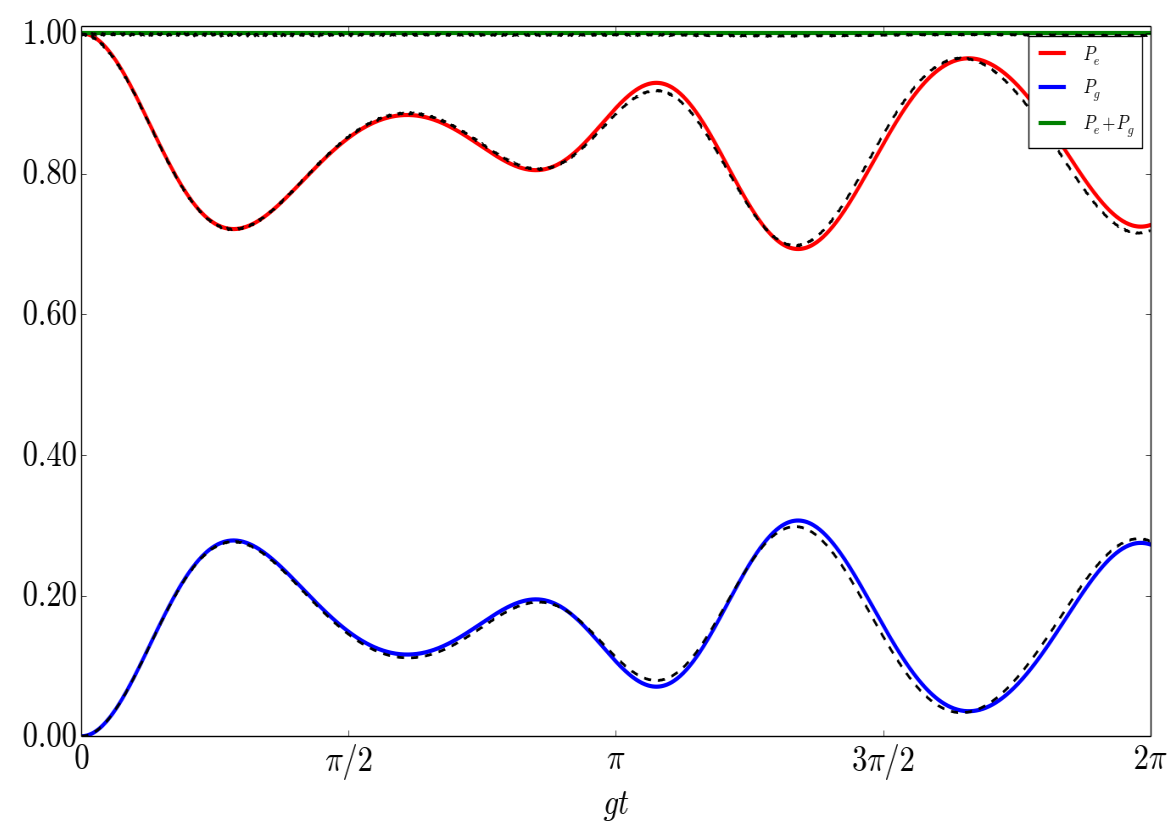

(b)

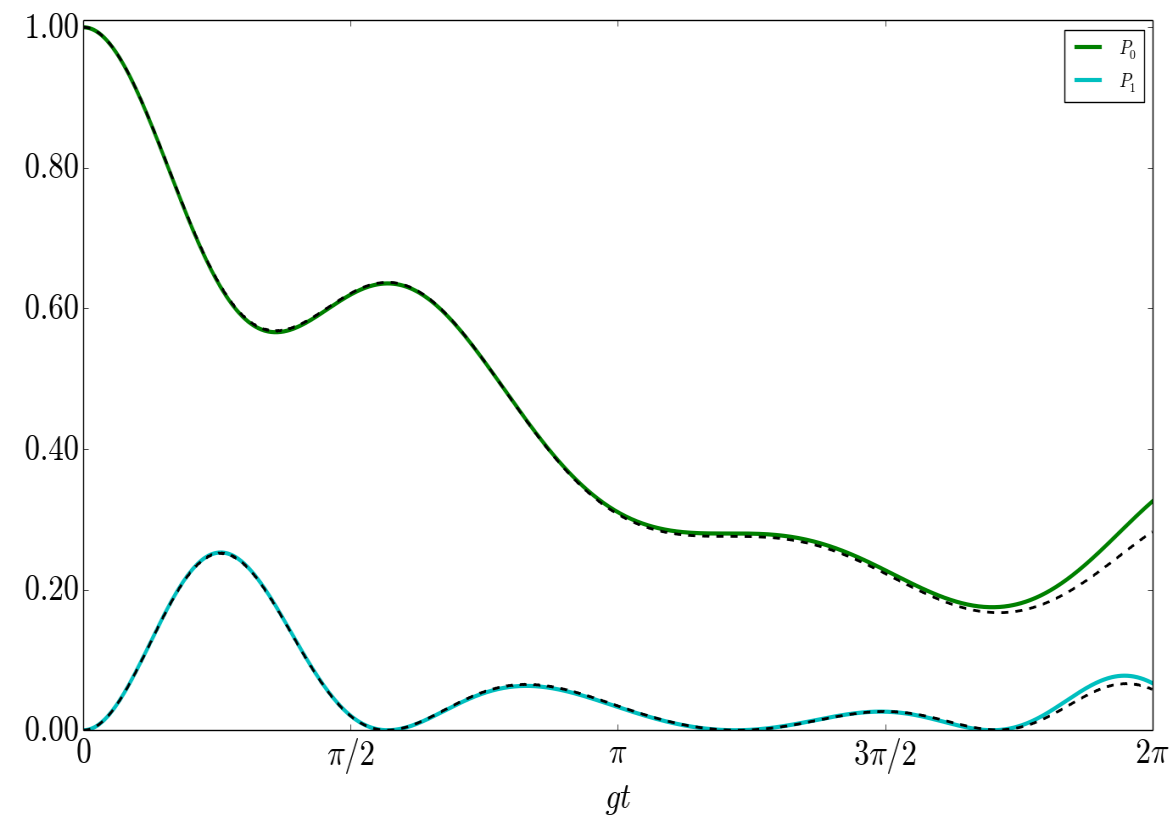

Fonte: Elaborada pelo autor. 


\subsection{Simulação do Zitterbewegung via EQC}

Os hamiltonianos efetivos das Eqs. (4.21) e (4.39) simulam matematicamente o Hamiltoniano de Dirac 2+1 D e 1+1 D em ausência de um potencial externo, respectivamente. Portanto, podemos estudar a dinâmica de uma partícula relativística livre por meio deles. Nesta seção demostraremos como simular o movimento oscilatório inesperado de uma partícula livre de Dirac (Zitterbewegung) em uma dimensão. Para analisar tal feito, reescrevemos a Eq.(4.39) como:

$$
H_{1 D}=\zeta_{+} \sigma_{3}+R_{a} \frac{\lambda_{a}^{2}}{\Delta_{a}} p_{1} \sigma_{1}
$$

por conseguinte, a equação de Schrödinger associada à Eq.(4.45) :

$$
H_{1 D}|\psi\rangle=i \hbar \frac{d|\psi\rangle}{d t}
$$

exibe a mesma dinâmica da equação de Dirac $1+1$ D para uma partícula relativística livre, na qual $|\psi\rangle$ representa um bispinor. Agora usando o $H_{1 D}$ determinamos o operador velocidade da partícula relativística livre com a seguinte expressão:

$$
\frac{d S_{1}}{d t}=\frac{i}{\hbar}\left[H_{2 D}, S_{1}\right]=R_{a} \frac{\lambda_{a}^{2}}{\Delta_{a}} \sigma_{1}
$$

por conseguinte a evolução temporal do operador posição da partícula depende da evolução de $\sigma_{1}$ na representação de Heisenberg. Além do mais, a relação (4.47) tem dois casos importantes dependendo do valor da massa da partícula relativística. No caso quando a partícula não tem massa obtemos que $\sigma_{1}$ é uma constante de movimento, dessa maneira, a evolução temporal do operador posição da partícula é análoga à evolução temporal da posição de uma partícula livre clássica, consequentemente neste caso não apresenta-se o Zitterbewegung. Para o caso quando a partícula é massiva, obtemos que $d \sigma_{1} / d t \neq 0$, portanto a evolução temporal do operador posição da partícula fica da seguinte forma (ver Cap.2.4):

$S_{1}(t)=S_{1}(0)+\left(R_{a} \frac{\lambda_{a}^{2}}{\Delta_{a}}\right)^{2} p_{1} H_{1 D}^{-1} t+\frac{i \hbar}{2} R_{a} \frac{\lambda_{a}^{2}}{\Delta_{a}}\left(\sigma_{k}(0)-R_{a} \frac{\lambda_{a}^{2}}{\Delta_{a}} p_{k} H_{1 D}^{-1}\right) H_{1 D}^{-1}\left[e^{-2 i H_{1 D} t / \hbar}-1\right]$.

Esta equação simula o Zitterbewegung com parâmetros típicos de EQC. Se considerarmos um estado bispinor $|\psi\rangle=\left|\psi_{\text {campo }}\right\rangle \otimes\left|\psi_{\text {atomo }}\right\rangle$, podemos estimar a frequência $\left(\omega_{z}\right)$ e amplitude $\left(A_{z}\right)$ do zitterbewegung relacionada à média do $\left\langle S_{k}(t)\right\rangle$ da seguinte maneira: 


$$
\begin{gathered}
\omega_{z}=\frac{2\left|\left\langle H_{1 D}\right\rangle\right|}{\hbar}=\frac{2}{\hbar} \sqrt{p_{0}^{2}\left(R_{a} \frac{\lambda_{a}^{2}}{\Delta_{a}}\right)^{2}+\left(\zeta_{+}\right)^{2}}, \\
A_{z}=\frac{\hbar}{2 m c}\left(\frac{m c^{2}}{\left\langle H_{1 D}\right\rangle}\right)^{2}=\frac{\hbar / 2\left(\zeta_{+} R_{a} \frac{\lambda_{a}^{2}}{\Delta_{a}}\right)}{p_{0}^{2}\left(R_{a} \frac{\lambda_{a}^{2}}{\Delta_{a}}\right)^{2}+\left(\zeta_{+}\right)^{2}},
\end{gathered}
$$

sendo $p_{0}$ o autovalor do operador $p_{k}$. Para um elétron relativístico real, os valores da frequência e amplitude deste efeito relativístico são $\omega_{z} \sim 10^{21} \mathrm{~Hz}$ e $A_{z} \sim 10^{-3} \AA$, respectivamente, por esse motivo, o Zitterbewegung é um efeito que está fora do atual alcance experimental. Em vista da flexibilidade do nosso sistema, são aceitos valores de frequência e amplitude de oscilação que estão no intervalo $\omega_{z} \sim\left(0-10^{3}\right) H z$ e $A_{z} \sim\left(0-10^{4}\right) \AA$, com $R_{a} \sim \mu m$, os quais permitem um ganho comparados aos valores aceitados no sistema proposto na referência (33), que estão no intervalo $\omega_{z} \sim\left(0-10^{6}\right) H z$ e $A_{z} \sim\left(0-10^{3}\right) \AA$.

Uma maneira interessante de ilustrar o Zitterbewegung é plotar o gráfico relacionado com $\left\langle S_{k}\right\rangle$ versus o tempo de interação $t$, para diferentes valores de massa da partícula relativística livre. Para gerar o gráfico $\left\langle S_{1}\right\rangle$ versus $t$ via cálculo numérico consideramos a correspondência que existe entre $\left\langle s_{1}\right\rangle$ e a quadratura do campo $R_{a}\left\langle a_{1}^{\dagger}+a_{1}\right\rangle$ de nosso sistema, onde mudamos o parâmetro $\zeta_{+}^{\prime}$ através da variação das forças de acoplamento, $\Omega_{1}, \Omega_{2}, \tilde{\Omega}_{1}$ e $\tilde{\Omega}_{2}$ no intervalo $\left(10^{4}-10^{5}\right) H z$. Além do mais, usamos $\Delta_{a}=20 \lambda$ e $\lambda_{a}=\lambda / 2$ o qual corresponde à simulação da velocidade da luz $c=1.25 R_{a} k H z$ (usada para todas as curvas). A medida do valor $\left\langle S_{1}\right\rangle$ é ilustrada na Figura (4.5), para a partícula inicialmente preparada no estado $|0\rangle \otimes|e+g\rangle / \sqrt{2}$. Nesta Figura a curva linear azul representa um partícula sem massa movendo-se na velocidade da luz, a qual é simulada em nosso sistema quando $m=\zeta_{+}^{\prime} /\left(R_{a} \frac{\lambda_{a}^{2}}{\Delta_{a}}\right)^{2} \sim 10^{-35} \mathrm{~kg}$. As outra curvas representam o acréscimo de massa da particula, com comprimento de Compton $\Lambda=h R_{a} \lambda_{a} / \zeta_{+}^{\prime} \Delta_{a}$.

O que nos demostra que o Zitterbewegung pode-se simular usando parâmetros típicos em experimentos de EQC. Agora para estudar esse efeito neste contexto, é necessário medir experimentalmente a média da quadratura do campo eletromagnético $\left\langle a_{1}^{\dagger}+a_{1}\right\rangle$. Uma proposta por Solano et al. (60) mostra que é passível obter informação das quadraturas do campo através dos estados eletrônicos de um átomo que interage com esse campo em uma cavidade. Com isso, é possível coletar as informações relacionados ao $\left\langle a_{1}^{\dagger}+a_{1}\right\rangle$ para plotar o gráfico ilustrada na Figura (4.5). 
Figura 4.5 - Valor esperado, $\left\langle S_{1}\right\rangle$, para uma partícula relativística com diferentes massas. A curva linear azul representa um partícula sem massa movendo-se na velocidade da luz. As outras curvas representam partículas massivas com $\Lambda_{1}=3.11 R_{a}, \Lambda_{2}=0.99 R_{a}, \Lambda_{3}=0.57 R_{a}$ e $\Lambda_{4}=0.30 R_{a}$, respetivamente:

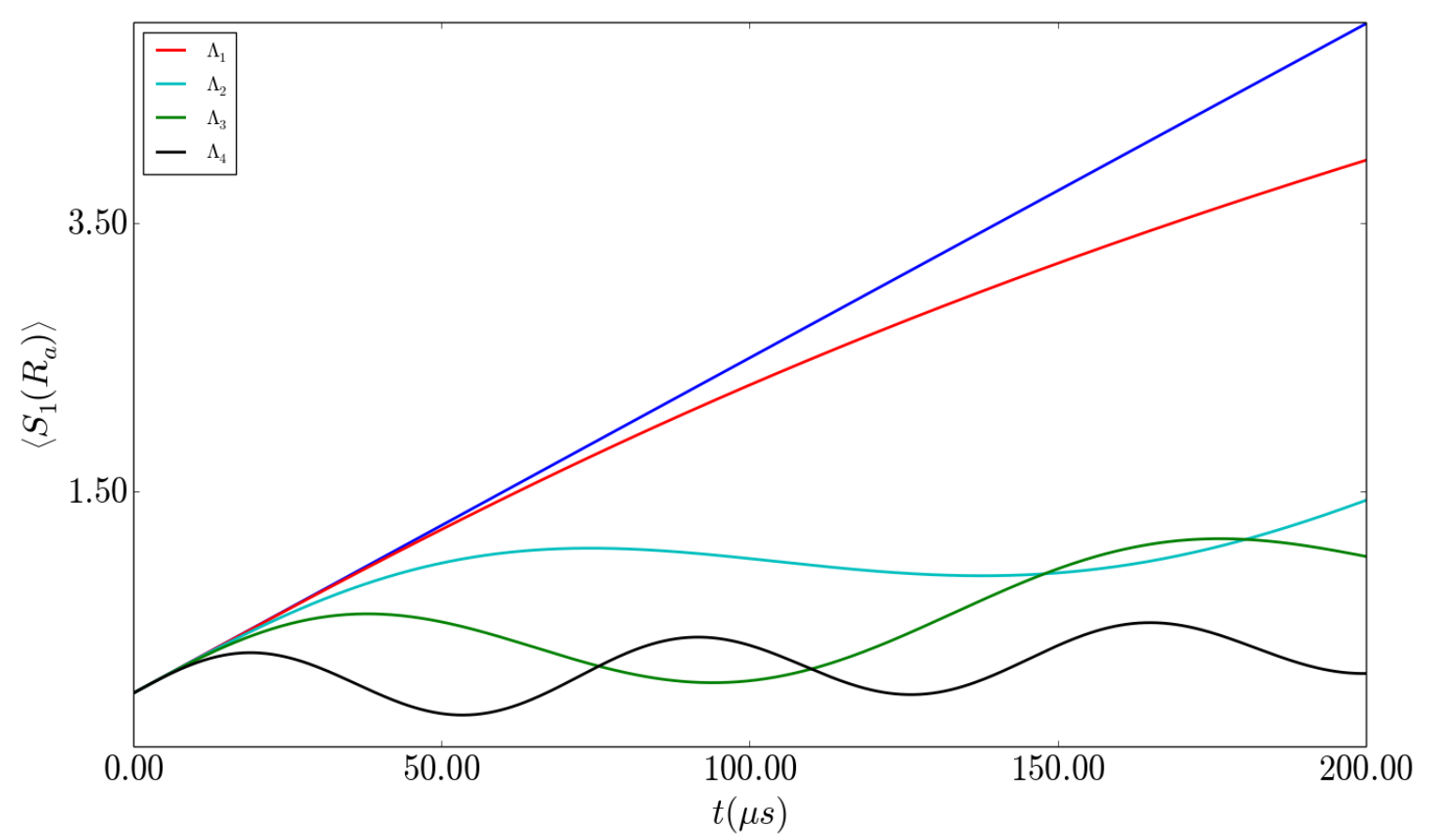

Elaborada pelo autor. 


\section{Capítulo 5}

\section{Conclusão}

Nesta dissertação abordamos alguns dos pontos de contato entre a Eletrodinâmica Quântica de Cavidades (EQC) e a Mecânica Quântica Relativística, estudando a chamada Simulação Quântica. Realizamos um estudo de dois sistemas específicos: um átomo de quatro níveis submetido a interações fora de ressonância com dois modos da cavidade e com quatro campos clássico; e um átomo de três níveis interagindo fora de ressonância com um modo da cavidade e com dois campos clássico. Nesses dois sistemas, partimos da engenharia de hamiltonianos no contexto específico em que as interações átomo-campo ocorrem no regime dispersivo. Obtidos os hamiltonianos efetivos desejados, em regime particulares dos parâmetros envolvidos, passamos a compará-los com os hamiltonianos de Dirac $2+1$ D e $1+1$ D. Concluímos que é possível simular no contexto de EQC a equação de Dirac 2+1 D e 1+1 D, já que os hamiltonianos efetivos apresemtam a mesma descrição matemática dos hamiltonianos de Dirac $2+1$ D e $1+1 \mathrm{D}$.

Além disso, simulamos o efeito zitterbewegung em uma dimensão manipulando com parâmetros típicos em EQC, os valores da velocidade da luz e a energia de repouso da partícula relativística livre simulada. Neste contexto mostramos que a amplitude e a frequência de oscilação da partícula simulada têm valores que são mais acessíveis experimentalmente que os valores da frequência e amplitude de oscilação deste efeito relativístico real cumpriendo com o objetivo da simulação quântica o qual é simular um sistema quântico de difícil acesso experimental através de outro com significativo grau de controle. consequentemente, concluímos que a dinâmica de um elétron livre no formalismo da mecânica quântica relativística, pode ser simulada usando experimentos em EQC.

Claramente, este trabalho não aborda todos aspectos da conexão entre a EQC e a Mecânica Quântica Relativística, de forma que algumas das perspectivas futuras desse trabalho situam-se em estudá-la 


\section{Referências}

1 MERZBACHER, E. Quantum mechanics. New York: John Wiley Sons, 1998. ISBN 9780471887027.

2 COHEN-TANNOUDJI, B. C. Quantum mechanics. Michigan: Wiley, 1991. ISBN 9780471164333.

3 SAKURAI, J.; NAPOLITANO, J. Modern quantum mechanics. Boston: Pearson Education, 2014. ISBN 9780321972071.

4 KLEIN, O. Quantentheorie und fundamentalnfdimensionale relativitatstheorie. Zeitschrift fur Physik, v. 37, n. 12, p. 895-906, 1926.

5 GORDON, W. Der comptoneffekt nach der schrodingerschen theorie. Zeitschrift fur Physik, v. 40, n. 1-2, p. 117-133, 1926.

6 DIRAC, P. A. M. The quantum theory of the electron. Proceedings of the Royal Society of London A, v. 117, n. 778, p. 610-624, 1928.

7 SAKURAI, J. J. Advanced quantum mechanics. Chicago: Addison-Wesley, 1967. ISBN 0201067102.

8 ANDERSON, C. D. The positive electron. Physical Review Letters, v. 43, n. 6, p. 491-494, 1933.

9 KLEIN, O. Die reflexion von elektronen an einem potentialsprung nach der relativistischen dynamik von dirac. Zeitschrift fur Physik, v. 53, n. 3-4, p. 157-165, 1929.

10 BREIT, G. An interpretation of Dirac's theory of the electron. Proceedings of the National Academy of Sciences of the United States of America, v. 14, n. 7, p. 553-559, 1928.

11 SCHRÖDINGER, E. Über die kräftefreie bewegung in der relativistischen quantenmechanik. Chicago: Akademie der wissenschaften in kommission bei W. de Gruyter u. Company, 1930.

12 ZAWADZKI, W.; RUSIN, T. M. Zitterbewegung (trembling motion) of electrons in semiconductors. Journal of Physics, v. 23, n. 14, p. 143201, 2011.

13 HUANG, K. On the zitterbewegung of the Dirac electron. American Journal of Physics, v. 20, n. 8, p. 479-484, 1952.

14 WALLS, D. F. Squeezed states of light. Nature, v. 306, n. 5939, p. 141-146, 1983. 
15 RAIMOND, J. M.; BRUNE, M.; HAROCHE, S. Manipulating quantum entanglement with atoms and photons in a cavity. Review of Modern Physics, v. 73, n. 3, p. 565-582, 2001.

16 LEIBFRIED, D. et al. Quantum dynamics of single trapped ions. Review of Modern Physics, v. 75, n. 1, p. 281-324, 2003.

17 CORNELL, E. A.; WIEMAN, C. E. Nobel lecture: Bose-Einstein condensation in a dilute gas, the first 70 years and some recent experiments. Review of Modern Physics, v. 74, n. 3, p. 875-893, 2002.

18 KETTERLE, W. Nobel lecture: When atoms behave as waves: Bose-Einstein condensation and the atom laser. Review of Modern Physics, v. 74, n. 4, p. 1131-1151, 2002 .

19 HAROCHE, S.; RAIMOND, J. Exploring the quantum. New York: Oxford University Press, 2013. (Oxford Graduate Texts). ISBN 9780199680313.

20 BRUNE, M. et al. Quantum Rabi oscillation: a direct test of field quantization in a cavity. Physical Review Letters, v. 76, n. 11, p. 1800-1803, 1996.

21 BRUNE, M. et al. Observing the progressive decoherence of the "meter" in a quantum measurement. Physical Review Letters, v. 77, n. 24, p. 4887-4890, 1996.

22 HAGLEY, E. et al. Generation of Einstein-Podolsky-Rosen pairs of atoms. Physical Review Letters, v. 79, n. 1, p. 1-5, 1997.

23 RAUSCHENBEUTEL, A. et al. Coherent operation of a tunable quantum phase gate in cavity qed. Physical Review Letters, v. 83, n. 24, p. 5166-5169, 1999.

24 BRATTKE, S.; VARCOE, B. T. H.; WALTHER, H. Generation of photon number states on demand via cavity quantum electrodynamics. Physical Review Letters, v. 86, n. 16, p. 3534-3537, 2001.

25 MONROE, C. et al. Demonstration of a fundamental quantum logic gate. Physical Review Letters, v. 75, n. 25, p. 4714-4717, 1995.

26 MEEKHOF, D. M. et al. Generation of nonclassical motional states of a trapped atom. Physical Review Letters, v. 76, n. 11, p. 1796-1799, 1996.

27 TURCHETTE, Q. A. et al. Deterministic entanglement of two trapped ions. Physical Review Letters, v. 81, n. 17, p. 3631-3634, 1998.

28 GREINER, M. et al. Quantum phase transition from a superfluid to a mott insulator in a gas of ultracold atoms. Nature, v. 415, n. 6867, p. 39-44, 2002.

29 NEELEY, M. A. M. Emulation of a quantum spin with a superconducting phase qudit. Science, v. 325, n. 5941, p. 722-725, 2009.

30 FRIEDENAUER, A. et al. Simulating a quantum magnet with trapped ions. Nature Physics, v. 4, n. 10, p. 757-761, 2008.

31 LEIBFRIED, D. et al. Trapped-ion quantum simulator: experimental application to nonlinear interferometers. Physical Review Letters, v. 89, n. 24, p. 247901, 2002. 
32 PORRAS, D.; CIRAC, J. I. Effective quantum spin systems with trapped ions. Physica Reviews Letters, v. 92, n. 20, p. 207901, 2004.

33 LAMATA, L. et al. Dirac equation and quantum relativistic effects in a single trapped ion. Physical Review Letters, v. 98, n. 25, p. 253005, 2007.

34 GERRITSMA, R. et al. Quantum simulation of the Dirac equation. Nature, v. 463, n. 7277, p. $68-71,2010$.

35 GERRITSMA, R. et al. Quantum simulation of the Klein paradox with trapped ions. Physical Review Letters, v. 106, n. 6, p. 060503, 2011.

36 ZHANG, X. Observing zitterbewegung for photons near the Dirac point of a two-dimensional photonic crystal. Physical Review Letters, v. 100, n. 11, p. 113903, 2008.

37 VAISHNAV, J. Y.; CLARK, C. W. Observing zitterbewegung with ultracold atoms. Physical Review Letters, v. 100, n. 15, p. 153002, 2008.

38 LEBLANC, L. J. et al. Direct observation of zitterbewegung in a Bose-Einstein condensate. New Journal of Physics, v. 15, n. 7, p. 073011, 2013.

39 QU, C. et al. Observation of zitterbewegung in a spin-orbit-coupled Bose-Einstein condensate. Physical Review A, v. 88, n. 2, p. 021604, 2013.

40 SCHLIEMANn, J.; LOSS, D.; WESTERVELT, R. M. Zitterbewegung. Physical Review Letters, v. 94, n. 20, p. 206801, 2005.

41 GREINER, W. Relativistic quantum mechanics. Berlin: Springer, 2000. ISBN 9783540674573.

42 GRIFFITHS, D. Introduction to elementary particles. Berlin: Wiley, 2008. ISBN 9783527618477.

43 CLOSE, F. Antimatter. New York: Oxford University Press, 2009. ISBN 9780191563966.

44 DiRAC, P.; DALITZ, R. The Collected Works of P. A. M. Dirac. New York: Cambridge University Press, 1995. ISBN 9780521362313.

45 DIRAC, P. A. M. The quantum theory of the emission and absorption of radiation. Proceedings of the Royal Society of London A, v. 114, n. 767, p. 243-265, 1927.

46 FERMI, E. Quantum theory of radiation. Review of Modern Physics, v. 4, n. 1, p. 87-132, 1932.

47 NESBET, R. K. Spontaneous emission in semiclassical radiation theory. Physical Review A, v. 4, n. 1, p. 259-264, 1971.

48 WENTZEL, G. Zur theorie des photoelektrischen effekts. Zeitschrift fur Physik, v. 40, n. 8, p. 574-589, 1926.

49 CRISP, M. D.; JAYNES, E. T. Radiative effects in semiclassical theory. Physical Review, v. 179, n. 5, p. 1253-1261, 1969. 
50 JAYNES, E.; CUMMINGS, F. Comparison of quantum and semiclassical radiation theories with application to the beam maser. Proceedings of the IEEE, v. 51, n. 1, p. 89-109, 1963.

51 KIM, J. I. et al. Classical behavior with small quantum numbers: the physics of ramsey interferometry of rydberg atoms. Physical Review Letters, v. 82, n. 24, p. 4737-4740, 1999.

52 FARAON, A. et al. Integrated quantum optical networks based on quantum dots and photonic crystals. New Journal of Physics, v. 13, n. 5, p. 055025, 2011.

53 SCULLY, M.; ZUBAIRY, S. Quantum optics. New York: Cambridge University Press, 1997. ISBN 9780521435956.

54 PIZA, A. D. T. Mecânica quântica. Sao Paulo: EDUSP, 2003. ISBN 9788531407482.

55 GERRY, C.; KNIGHT, P. Introductory quantum optics. New York: Cambridge University Press, 2005. ISBN 9780521527354.

56 KNIGHT, P.; MILONNI, P. The Rabi frequency in optical spectra. Physics Reports, v. 66, n. 2 , p. $21-107,1980$.

57 JAMES, D. F.; JERKE, J. Effective hamiltonian theory and its applications in quantum information. Canadian Journal of Physics, v. 85, n. 6, p. 625-632, 2007.

58 ZHENG, S.-B.; GUO, G.-C. Efficient scheme for two-atom entanglement and quantum information processing in cavity qed. Physical Review Letters, v. 85, n. 11, p. 2392-2395, 2000.

59 CELERI, L. et al. Switching off the reservoir through nonstationary quantum systems. Journal of Physics B, v. 41, n. 8, p. 085504, 2008.

60 LOUGOVSKI, P. et al. Instantaneous measurement of field quadrature moments and entanglement. European Physical Journal D, v. 38, n. 3, p. 423-426, 2006. 